\title{
A statistical-coupled model for organic-rich shale gas transport
}

\author{
Gaohui Cao ${ }^{\mathrm{a}, \mathrm{b}}$, Mian Lin ${ }^{\mathrm{a}, \mathrm{b}, *}$, Wenbin Jiang ${ }^{\mathrm{a}}$, Wenlong Zhao ${ }^{\mathrm{a}, \mathrm{b}}$, Lili $\mathrm{Ji}^{\mathrm{a}}$, Caoxiong $\mathrm{Li}^{\mathrm{a}}{ }^{\mathrm{b} b}$, Da Lei ${ }^{\mathrm{c}}$ \\ a Institute of Mechanics, Chinese Academy of Sciences, Beijing, 100190, China \\ b School of Engineering Science, University of Chinese Academy of Sciences, Beijing, 100049, China \\ ${ }^{\mathrm{c}}$ School of Chemical Engineering and Technology, Xi'an Jiaotong University, Xi'an, Shaanxi, 710049, China
}

\section{A R T I C L E I N F O}

\section{Keywords:}

Shale gas

Multiscale

Permeability

3D coupled model

oREV

\begin{abstract}
A B S T R A C T
Computation of shale permeability is challenging owing to the complicated physics of gas flow and the smallscale heterogeneity in nanometer pores. In this study, a statistical-coupled model (SCM), based on the combination of FIB/SEM and SEM imaging measurements and statistical analysis is proposed to bridge the nanoporescale with the organic representative elementary volume (oREV)-scale. FIB/SEM imaging is adopted to get the nanometer pores properties in organic matter (OM), and SEM imaging is used to get the OM content distribution. With the analysis of nanopores of a Longmaxi shale sample from the Chongqing Province, China, it is demonstrated that the pore size distribution obtained from FIB/SEM images of typical samples is representative considering the principal parts of the pore radii are similar comparing with 2D SEM image. Then, the SCM is constructed based on the combination of the statistical method, the series-parallel model and the equivalent model for microstructures, and the rationality of SCM are also investigated. The obtained characteristic parameters show an excellent performance in calculating the SCM element permeability with a small deviation of less than $3 \%$ and a significantly faster computation speed comparing with the previous literature by approximately 400 times. Using the SCM, a method for the construction of oREV and the determination of oREV-scale permeability for the organic-rich shale is presented. Finally, the sensitivity analyses of oREV-scale permeability are conducted and the results show that the permeability is sensitive to the OM content distribution, the district of shale sample and the permeability of IOM. The influence of OM permeability on macroscale is also analyzed. The new model can advance the understanding of the multiscale phenomena and establish a relationship between microscale properties and macroscale behavior.
\end{abstract}

\section{Introduction}

Organic-rich shale has attracted significant attention to meet world's increasing energy demand. Differing from conventional reservoirs, gas shale, is rich in nanopores with extremely low porosity and permeability. Special techniques are necessary to be used in the gas exploitation for the unconventional reservoirs. For a shale reservoir, hydraulic fracturing is widely used which causes a complex multiscale system for gas transport (Chen et al., 2015b; Osiptsov et al., 2017; Yang et al., 2017; Zhiming et al., 2014). And the strong heterogeneity of organic matter (OM) and inorganic matter (IOM) in shale also adds significant complexities into the multiscale problem (Yan et al., 2018). The macroscopic properties of shale such as porosity, permeability and adsorption are dependent on its micro-structures, components and flow regimes. It is of significant importance to get the properties of the representative elementary volume (REV) which serves as the bridge of microscale and macroscale. Over several years since the concept of REV was floated by Bear, it is known that REV is the volume at which the averaging of the reservoir attribute becomes stable (Bear, 1972). An REV can be defined for each porous medium property or system condition of interest such as porosity, moisture saturation, permeability (Costanza-Robinson et al., 2011). In recent years, several approaches are proposed for determining the REV. Lake and Srinivasan (2004) presented a method to determine the REV of an attribute from a log-log plot of the variance of attribute's linear average vs. length scale. Singh et al. proposed a sophisticated approaches to estimate REV using variance-based approach (Singh, 2014, 2017; Singh and Srinivasan, 2014). It is hypothesized that accurate assessment of the scaling characteristics of reactive-transport processes in reservoirs requires analysis of the spatiotemporal variability of the underlying rock properties of the reservoir. A semi-analytical model considering both the spatial and temporal characteristics of the reservoir attribute is used to derive the REV of attribute in combined space and time. Naraghi and Javadpour (2015) compared the sensitivity of the total apparent permeability to the local heterogeneity by changing the sample size. They calculated the mean permeability and the standard deviation of several stochastic

\footnotetext{
* Corresponding author. Institute of Mechanics, Chinese Academy of Sciences, Beijing, 100190, China.

E-mail address: linmian@imech.ac.cn (M. Lin).
} 


\begin{tabular}{|llll|}
\hline \multicolumn{2}{|l|}{ Nomenclature } & $t$ & time \\
& & $T$ & absolute temperature, $\mathrm{K}$ \\
$d_{m}$ & diameter of a gas molecule & $V_{a b s, s t p}$ & volume of gas adsorbed at STP \\
$D_{f}$ & surface fractal dimension & $V_{L}$ & ultimate adsorption amount \\
$K_{\text {iom }}$ & permeability of IOM & $Z$ & compressibility factor \\
$K_{o m}$ & permeability of OM & $\alpha$ & TMAC \\
$K_{d, o m}$ & absolute permeability of OM & $\tau$ & tortuosity \\
$K_{e}$ & permeability of the SCM element & $\mu_{g}$ & gas viscosity \\
$K_{s}$ & permeability of SCM & $\rho$ & gas density \\
$M$ & molecular molar mass of methane & $\rho_{s}$ & shale density \\
$P$ & pressure & $\Psi$ & OM content \\
$p_{L}$ & pressure when adsorption reaches half of the ultimate & $\mu, \sigma$ & characteristic parameters of the OM content distribution \\
& adsorption amount & $\Phi_{s}$ & porosity of shale matrix \\
$R$ & universal gas constant & $\Phi_{f}$ & flowing porosity \\
& & &
\end{tabular}

realizations to determine the REV when the standard deviation goes approximately to zero. In this study, the REV is defined for the volume that is sufficient to involve the heterogeneity caused by organic matter, referred to as organic REV (oREV). As the flow scale in shale spans widely, a reasonable approach for its multiscale modeling involves obtaining the oREV-scale permeability from a micro- or pore-scale and then followed by substituted into larger scale simulators, which can convert the complex multiscale phenomena into a computer-tolerable simulation model. Recently, numerous researchers have noticed the superior performance of multiscale models and applied them to investigate gas flow in shale (Akkutlu et al., 2017; Cao et al., 2017; Chen, 2016; Geng et al., 2016; Jiang et al., 2017; Singh and Javadpour, 2016; Song et al., 2016; Talonov and Vasilyeva, 2016).

In gas shale reservoirs, gas is stored in interconnected pore space or adsorbed onto $\mathrm{OM}$ and often sourced from the same reservoir rock (Darabi et al., 2012). From focused ion beam scanning electron microscope (FIB/SEM) images, it is obvious that there are abundant nanopores in OM. Gas flow in the nanopores is of significant nonlinear behaviors such as slip effect, Knudsen diffusion and adsorption. And the permeability equation proposed by Javadpour (2009) which considers the slip and Knudsen diffusion is widely used. In 2013, Mehmani et al. (2013) implemented Javadpour's equation for the throats of pore network model (PNM), then calculated the permeability of the entire pore network. However, using PNM in the whole region of shale is unsuitable: in IOM, the pores are highly dispersed and the main flow channel consists of natural and hydraulic fractures; while in OM, though the pores form pore network as the main gas storage space, the OMs are dispersed in IOM and isolated from one another (Cao et al., 2017). Natural gas production from shale formations involves highly complex geological features consisting of OMs embedded spatiallydistributed in IOM. Based on the different characteristics of $\mathrm{OM}$ and IOM in shale, treating them separately in a model has become a recognized idea, and there are two categories of models as follows.

One category is based on abstract or direct modeling on physical spatial images. Chen (2016) proposed a model on the basis of advanced, multiscale CT and SEM imaging of shale samples. The study related pore-scale information to larger-scale processes and applied sensitivity analysis and uncertainty quantification to reduce system complexities by characterizing the critical parameters. But it has limitations in the upscaling process with no consideration of the influence of $\mathrm{OM}$ on the macroscale permeability. Akkutlu and Fathi (2012) performed a study on a multiscale dual-porosity model with local kerogen heterogeneity by separating kerogen from inorganic matrix and representing both of them using continuum. In 2015, Akkutlu et al. (2015) adopted multiscale asymptotic analysis method to solve an one-dimensional dualporosity continuum mode. The study obtained the macroscopic parameters with the solutions to the cell problem defined in representative volume elements, which takes the fine-scale variations into account and average their effects on the macroscale. Follow Akkutlu's work, in 2016,
Talonov and Vasilyeva (2016) modeled shale by generating 2D coarse and fine grids on the SEM images, and utilized numerical homogenization technique to bridge the local problems to macro parameters. However, 2D model is used without considering the 3D heterogeneity and the continuum-scale simulator also needs the parameters obtained from pore-scale information. Besides, Jiang et al. (2017) proposed a 3D effective cluster-scale model to determine the characteristic parameters of the pores and the gas transport features in the OMs of shale. The study bridged the pore-scale morphology to cluster-scale parameters for OM based on the nanometer-resolution FIB/SEM images in conjunction with a high-precision pore network extraction algorithm (Axis \& Ball algorithm, AB (Yi et al., 2017)). Later, Cao et al. (2017) built a 3D coupled model of OM and IOM based on FIB/SEM images. PNM was utilized to represent the nanoporous $\mathrm{OM}$ to capture the complicated

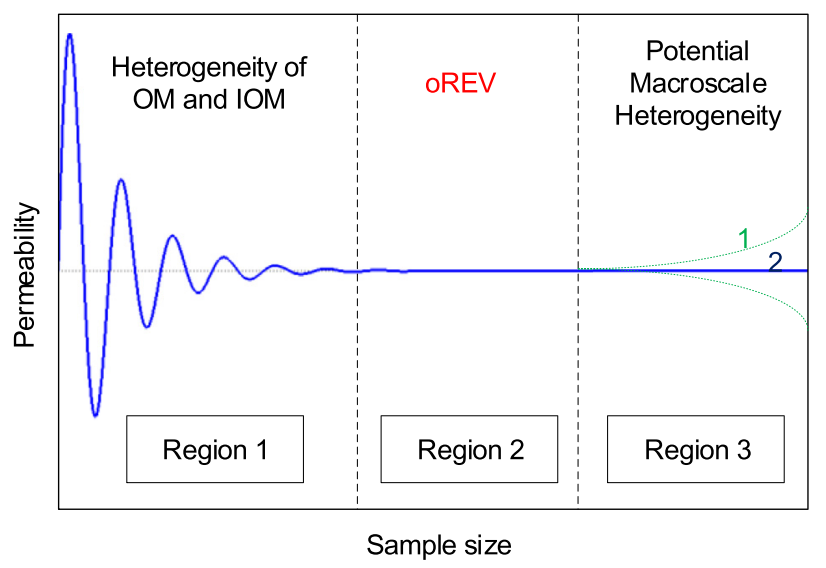

(a)

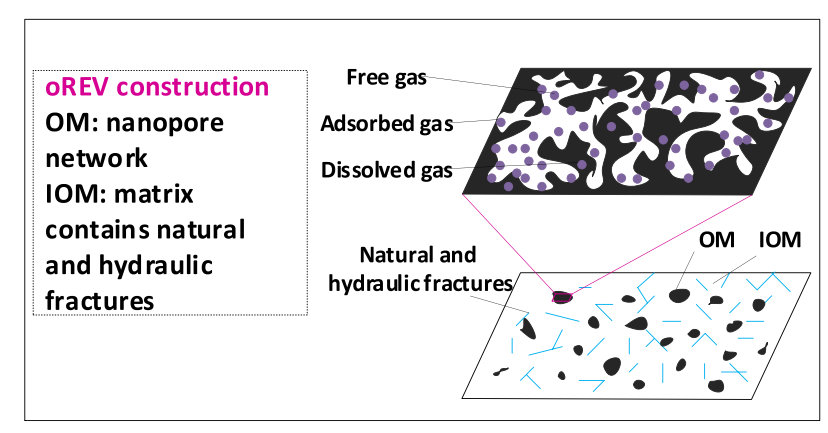

(b)

Fig. 1. Definition of oREV. (a) oREV in the multiscale system in shale, (b) oREV construction. 


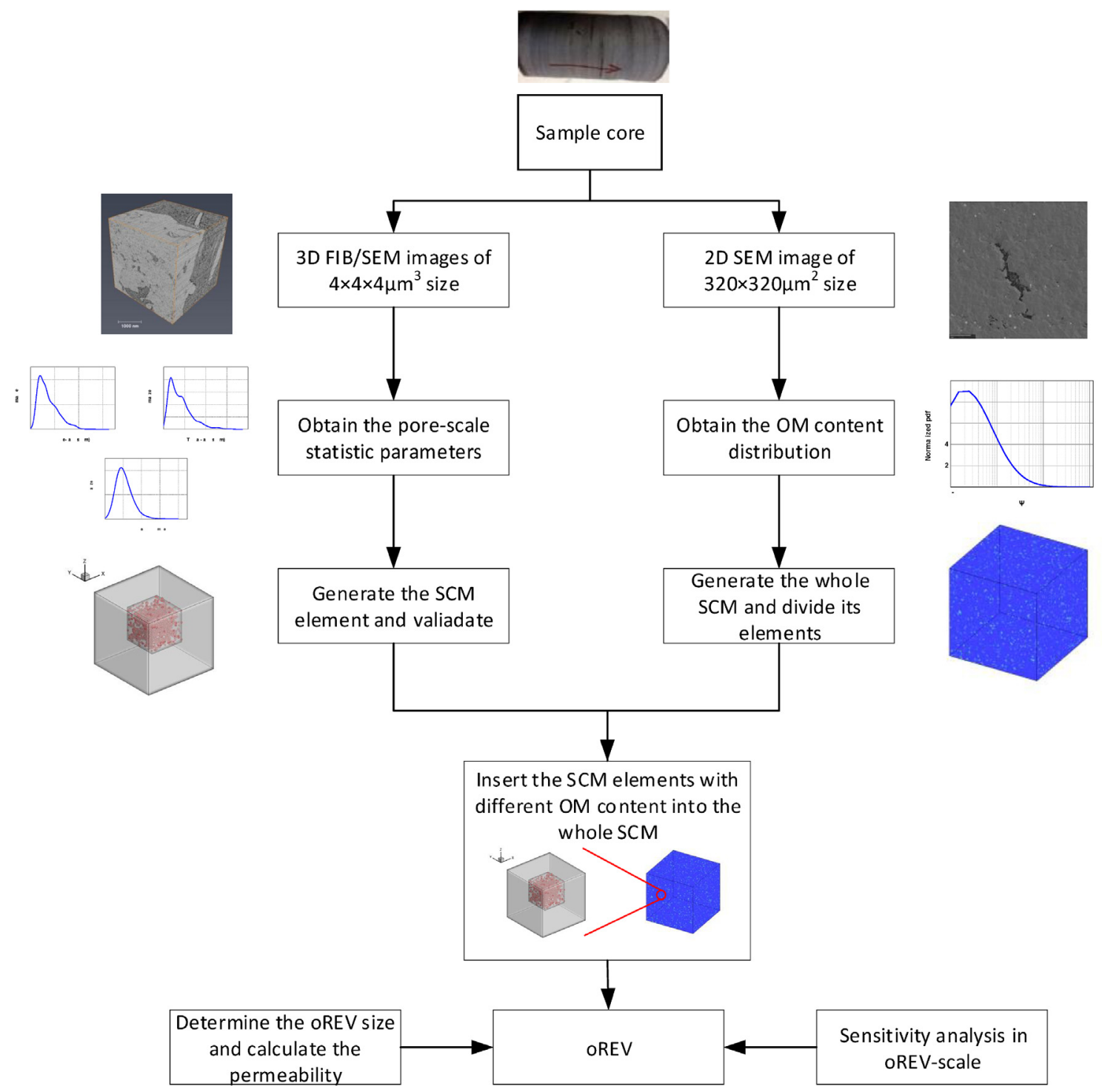

Fig. 2. Flowchart of the statistical-coupled model.

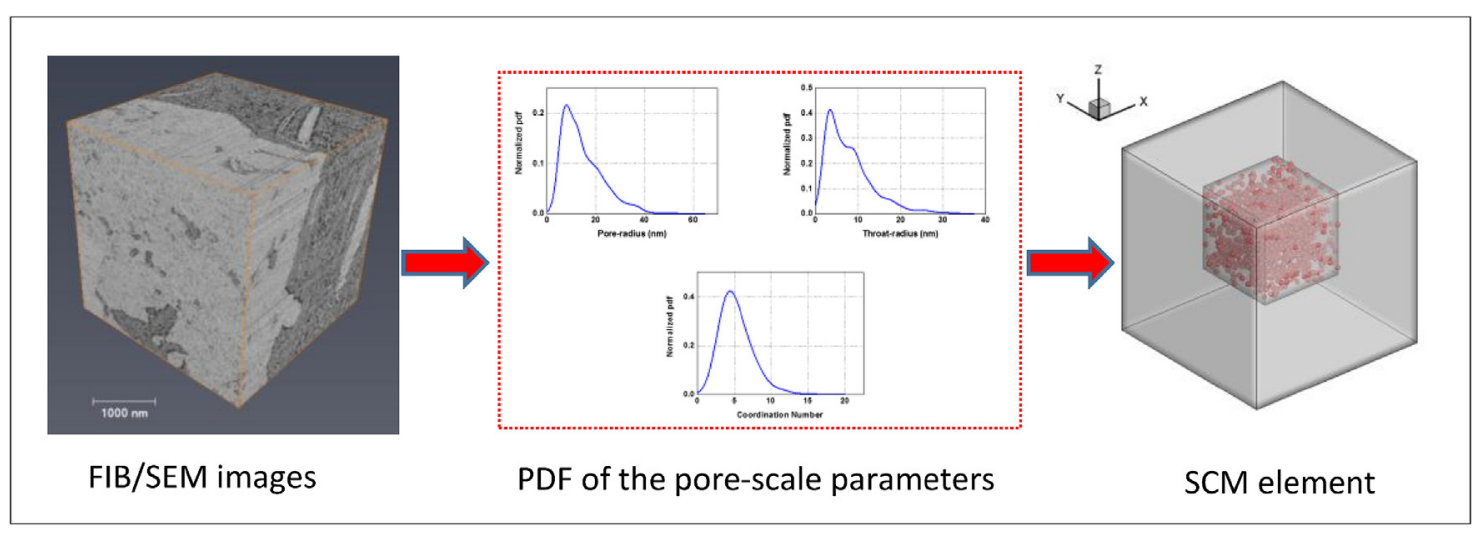

Fig. 3. Development procedure of the SCM element.

pore characteristics and spatial topology within it, while the interaction among IOM and fractures was homogenized as a single continuum medium and modeled by finite volume method (FVM). The method is applicable to capture the detailed interactions between OM and IOM in the 3D space with the size of several micrometers. However, the model is computationally limited to small domain size, accurate macroscale modeling requires simulating under reasonable simplification. Additionally, since the obtained information from the high-resolution images is usually highly localized, measurements in larger scale should also be involved.

Another category based on the statistics of microscale parameters. Among them, plenty of works using average pore size into the 


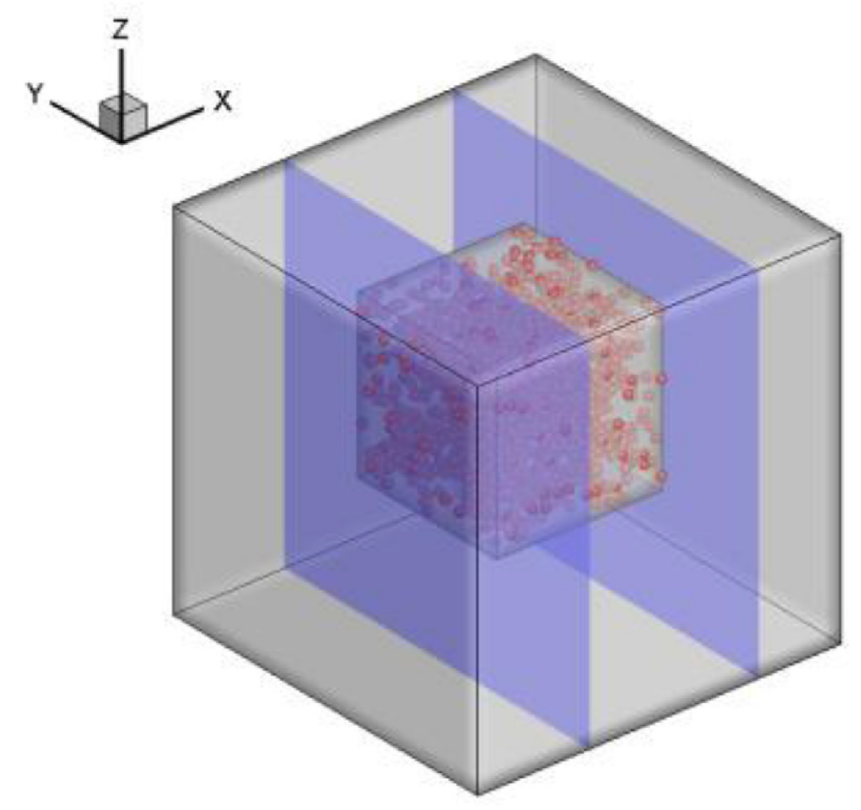

Fig. 4. Schematic of the permeability calculation of the SCM element based on the series-parallel model. The coupled model is divided into three parts with the two blue cross sections and the OM block is entirely within the middle part of the coupled model. (For interpretation of the references to colour in this figure legend, the reader is referred to the Web version of this article.)

permeability calculation of a porous medium to involve different flow regimes (Civan et al., 2011; Javadpour, 2009; Klinkenberg, 1941; Song et al., 2016). Considering the heterogeneity of OM and IOM, Naraghi and Javadpour (2015) applied Expectation-Maximization algorithm (EM) to separate pore size distributions of OM and IOM measured by nitrogen-adsorption experiments. They calculated the permeability of shale in representative equivalent size using an ensemble-based stochastic model. Results show that although the method is stochastic, it does not suffer much from uncertainty in permeability calculation. After that, Wang et al. (2016) employed EM algorithm to separate the pore size distribution of OM and IOM, then adopted the dusty gas model (DGM) and generalized Maxwell-Stefan model (GMS) to calculate the apparent permeability of the $2 \mathrm{D}$ shale matrix with $16 \times 8 \mu \mathrm{m}^{2}$ size. The study investigated on the distribution of OM and IOM and found it has a negligible influence on apparent permeability. However, the models have limitations in inserting pore-size distribution measured by the 3D experiment into a 2D model, and the tortuosity and the fractal dimension of the pore surface are determined by macroscale pulse-decay experiments.

As shown above, the permeability of shale can be calculated through modeling in representations of real physical space or using

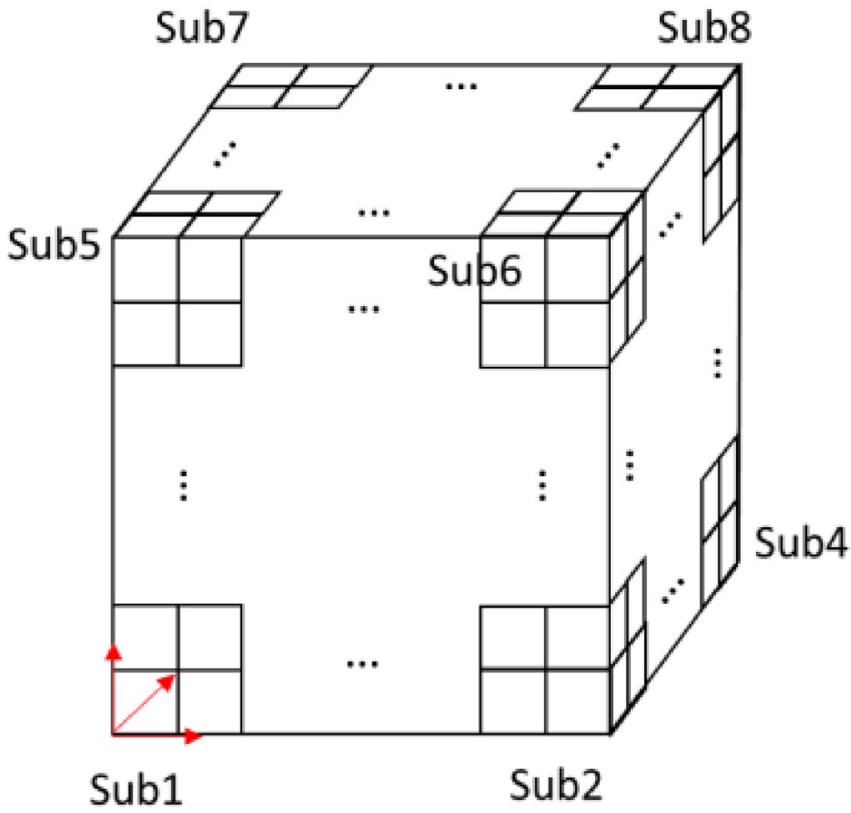

Fig. 6. Determination of oREV size. Eight sub-blocks of the SCM are generated beginning from the vertexes and oREV size is determined when the relative deviation of their permeability approaches zero.

statistical information. Among them, models using 2D simulation can hardly describe the shale characteristics integrally with no consideration of the 3D structures and heterogeneity. While the previous 3D models based on FIB/SEM are visually and computationally limited. It is necessary to develop a 3D model which is able to reflect the gas transport feature in the system of OM and IOM and obtain the dominant characteristic parameters from the nanopore-scale information then propagated to oREV-scale simulation.

In this study, we propose a statistical-coupled model (SCM) to bridge nanopore-scale and oREV-scale shale gas transport. Multiscale measurements are adopted in model, such as 3D FIB/SEM imaging with high-resolution for nanopore-scale information and 2D SEM imaging with larger field of view for $\sim 100 \mu \mathrm{m}$ heterogeneity. Based on the 3D structures from FIB/SEM images, reasonable equivalence is conducted by neglecting the OM/IOM distribution within the range of several micrometers which are not influential to model output, and the simulation proves high-efficiency using a newly proposed equation based on series-parallel model. And then, a larger SEM image is employed to build a whole SCM conditioned to the OM content distribution to find the oREV size. The permeability of oREV-scale shale is thus calculated and then its sensitivities are also analyzed. With the oREV-scale permeability, the influence of the microstructures on the macroscale behavior is assessed.

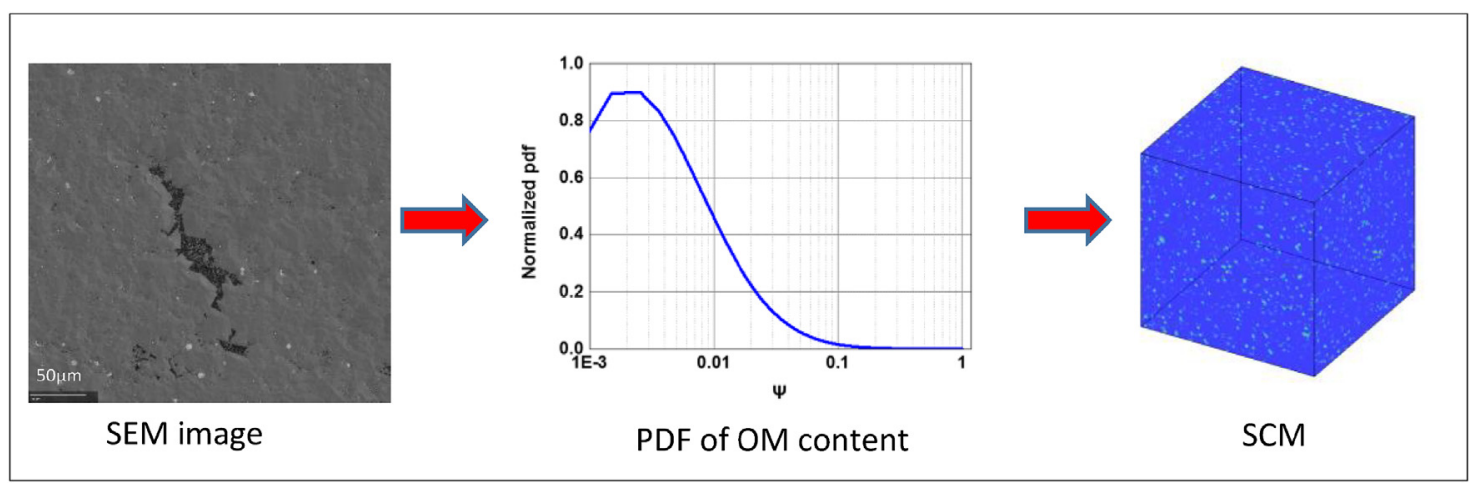

Fig. 5. Establishment method of SCM. SEM image is used to get the normalized PDF of the OM content and then construct the SCM. 


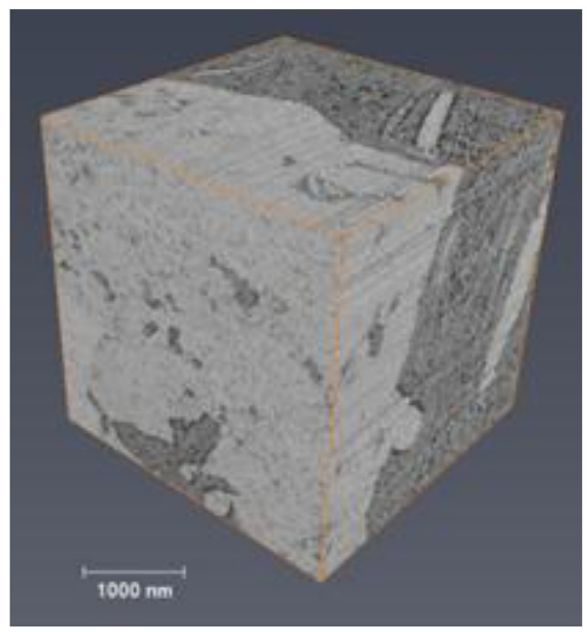

(a)

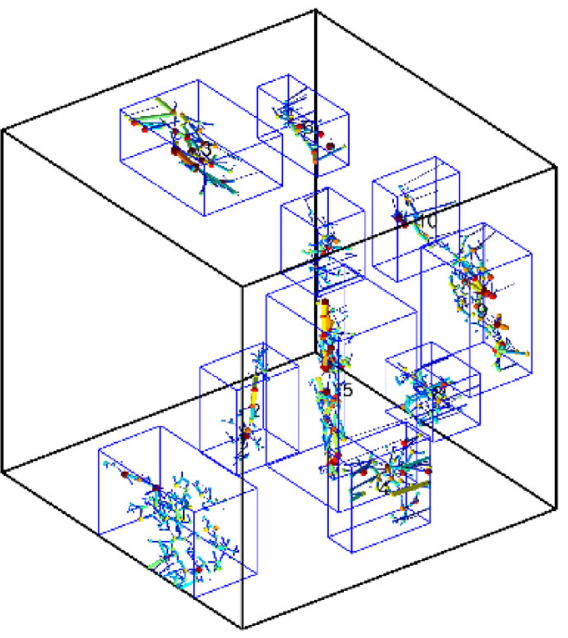

(b)

Fig. 7. (a) FIB/SEM images, (b) PNMs extract from the FIB/SEM images.

Table 1

Parameters of the extracted PNMs.

\begin{tabular}{lllllll}
\hline PNM number & $N_{p}$ & $N_{t}$ & $C N_{\text {avg }}$ & $R p_{\text {avg }}(\mathrm{nm})$ & $R t_{\text {avg }}(\mathrm{nm})$ & $D_{f}$ \\
\hline 1 & 280 & 300 & 2.14 & 13.27 & 7.42 & 2.17 \\
2 & 51 & 53 & 2.08 & 14.52 & 7.35 & 2.17 \\
3 & 79 & 83 & 2.10 & 11.93 & 6.26 & 2.19 \\
4 & 103 & 124 & 2.41 & 16.88 & 9.35 & 2.24 \\
5 & 124 & 125 & 2.02 & 15.74 & 8.04 & 2.19 \\
6 & 203 & 233 & 2.30 & 16.28 & 9.12 & 2.19 \\
7 & 47 & 47 & 2.00 & 15.12 & 7.57 & 2.15 \\
8 & 67 & 66 & 1.97 & 13.01 & 7.51 & 2.21 \\
9 & 106 & 105 & 1.98 & 14.06 & 7.43 & 2.17 \\
10 & 181 & 194 & 2.14 & 14.24 & 7.68 & 2.12 \\
\hline
\end{tabular}

$N_{p}$ and $N_{t}$ are the numbers of pores and throats in the pore network, respectively. $C N_{\text {avg }}$ is the average coordination number. $R p_{\text {avg }}$ is the arithmetic mean pore radius. $R$ th $_{\text {avg }}$ is the arithmetic mean throat radius.

This paper is organized as follows:Section 2 presents the frame of the research. In Section 3, the establishment method and the calculating principle of the new model are presented. In Section 4, a shale sample from the Longmaxi Formation from Chongqing Province, China is employed to construct the model and analyze its deviation, computational efficiency and uncertainty. In Section 5, the oREV size is determined, and the sensitivity analyses of the model in oREV-scale are conducted. The influence of microscale parameters on gas recovery is analyzed and the properties of two different shale samples are also evaluated. Finally, the conclusions of this work are drawn in Section 6.

\section{Research frame}

Unlike conventional reservoirs, in shale, hydraulic fracturing is a critical technique for gas production, and there are many natural and hydraulic fractures in IOM. The permeability of shale is primarily relevant to the relatively large pore and fractures in IOM, but the molecular behaviors (diffusion transport and nonlinear adsorption) relevant to the pores in OM (Akkutlu and Fathi, 2012). The OMs can be hardly seen in a quite large domain such as cores or reservoirs. However, highresolution imaging techniques such as FIB/SEM and SEM make it possible to observe the microscopic structures of OMs. In this study, we proposed a conception of oREV for shale permeability, as shown in Fig. 1. oREV is achieved when the volume is sufficient to involve the heterogeneity of IOM and OM. The permeability at the oREV-scale or larger will be constant and scale independent. Indeed, there will be potential macroscale heterogeneity in Region 3, which mainly caused by the heterogeneity of hydraulic fractures, such as Type 1 in Fig. 1a. In this study, our focus is on the microscale heterogeneity, and a simple type with a homogeneous spatial distribution of fractures (Type 2) is supposed for the macroscale simulation. oREV can determine the macroscopic parameters from microscopic measurements and be used to analyze the influence of microstructures on macroscale.

The determination of oREV-scale permeability of shale has proven difficult as: (1) because of the existence of very small pores (in nanoscale) in OM, using conventional approaches (such as Darcy's law) for fluid flow is challenging, (2) the gas pathway in shale is a complex system of OM and IOM which have different pore features and flow

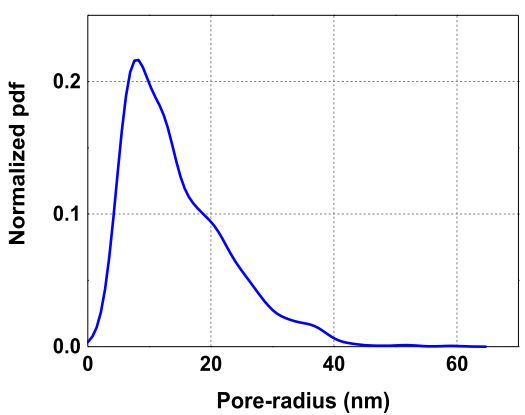

(a)

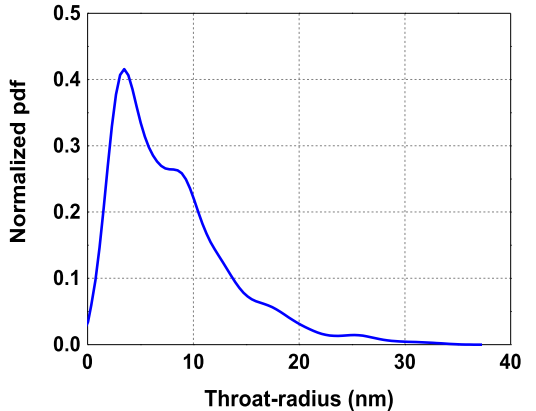

(b)

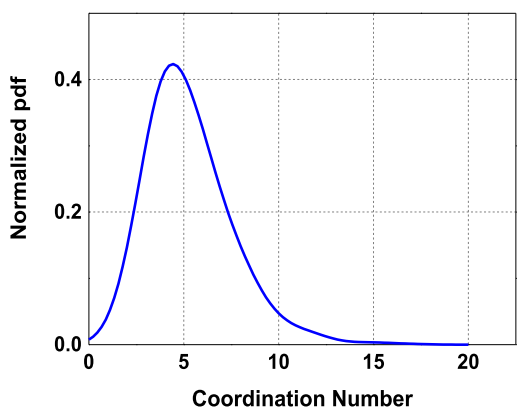

(c)

Fig. 8. Distributions of OM parameters, normalized PDF of (a) pore-radius, (b) throat-radius, and (c) coordination number. 


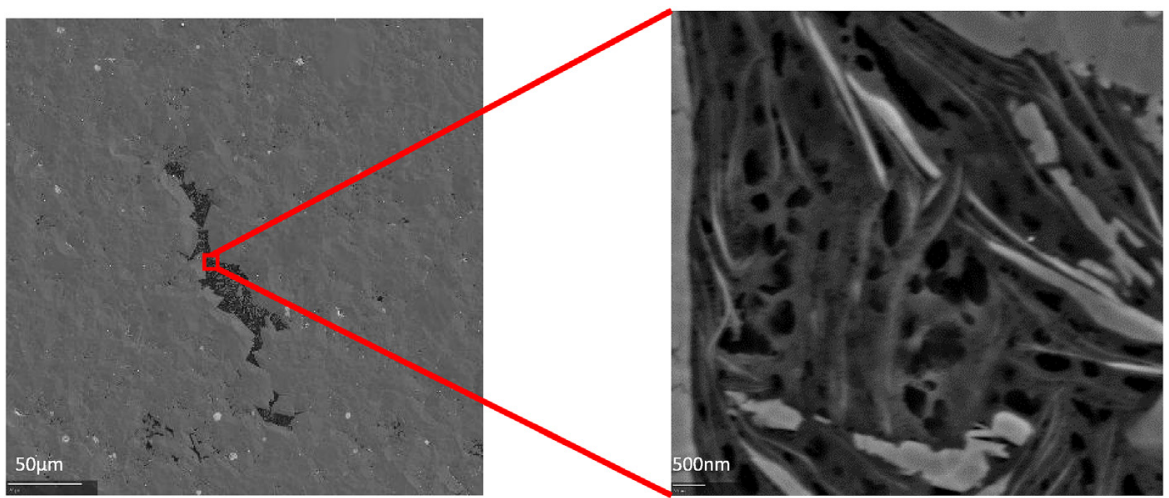

(a)

(b)

Fig. 9. Nanopores in SEM. (a) SEM image, (b) one of the OM blocks in the SEM image.

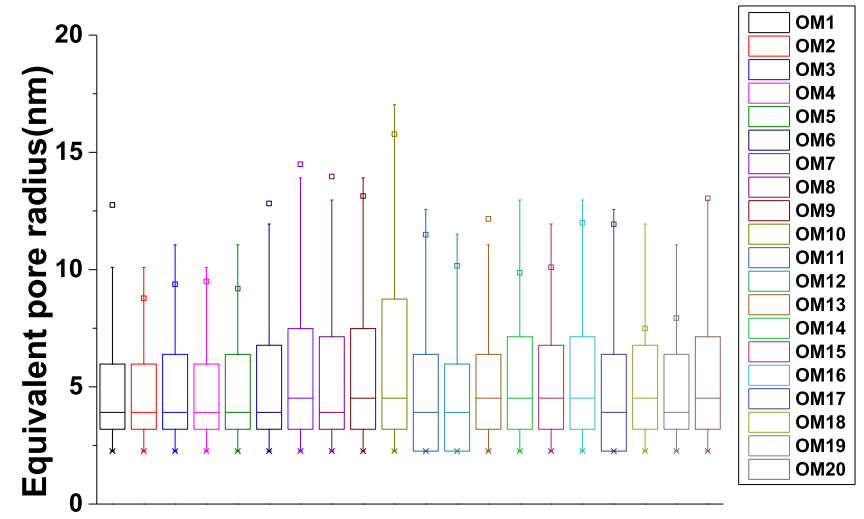

Fig. 10. Equivalent pore radii of the OMs in the SEM.

patterns, (3) shale has strong heterogeneity with a permeability significantly varies in different scales, and (4) though multiscale measurements can get plenty of information in shale, how to synthesize those information and extract the critical parameters remains difficult. In nanopore-scale, nanometer-resolution imaging technique, FIB/SEM imaging is a method to capture the 3D fundamental geometrical and transport properties. In our previous study (Cao et al., 2017), we built a $3 \mathrm{D}$ coupled model of OM and IOM based on FIB/SEM images. However, when it comes to the oREV-scale, the FIB/SEM imaging is insufficient as: (1) FIB/SEM imaging is limited to microscale with high expense and time-consuming (for example, a $4 \times 4 \times 4 \mathrm{~m}^{3}$ sample needs about $32,000 \mathrm{RMB}$ and $14 \mathrm{~h}$ in China), (2) an extremely detailed characterization of a small sample cannot involve larger-scale heterogeneity, and (3) the computing complexity will be extremely large though one can

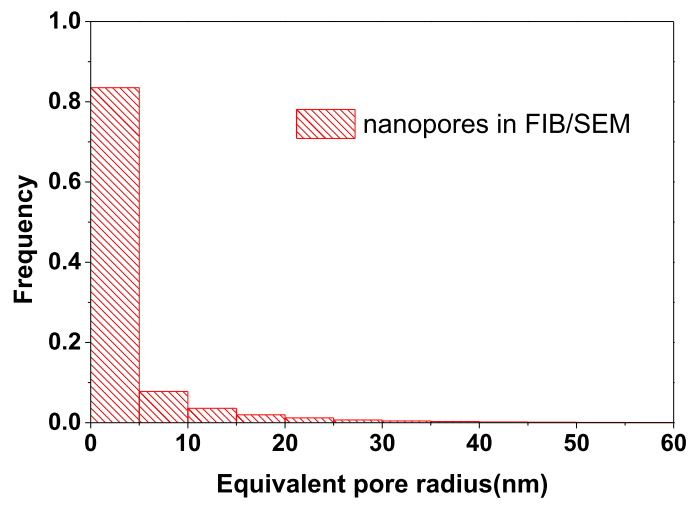

get a 3D model with $\sim 100 \mu \mathrm{m}$ length and $\sim \mathrm{nm}$ resolution through other methods. So measurement other than FIB/SEM should be involved to take consideration of the heterogeneity in larger sizes. In this study, SEM image with the size of about $320 \times 320 \mu \mathrm{m}^{2}$ is used to construct a model big enough for oREV searching. And the FIB/SEM images are used to obtain the 3D nanopore-scale properties of the model. Additionally, the statistical method is also introduced into the multiscale modeling, as it can no longer focus on the characterization and calculation accuracy of a small individual part but pays more attention to the statistical result of the whole parts with certain degree of microscopic similarities.

The research frame in this study is as follows: firstly, we analyze the properties of the nanopore based on the FIB/SEM and SEM images and find that the average pore-radius, throat-radius and coordination number of different OM blocks are basically the same. Results in Cao et al. (2017) have showed that the spatial distribution of OM/IOM has a weak effect on microscale permeability. And thus, the SCM element is established by neglecting the week-influence factors such as the distribution of OM/IOM and homogenizing some parameters such as the fractal dimension of the pore surface. In the SCM element, the OMs which disperse in the IOM are integrated into one block to get an equivalent result. Through reasonable equivalence without changing the distribution of characteristic parameters (pore-radius, throat-radius and coordination number), the error caused by the integration is small and will be analyzed in Section 4. Finally, the overall SCM is formed conditioned to the distribution of OM content based on SEM. The SCM is composed of the SCM elements which contain different OM content randomly selected from the OM content distribution. Using the SCM, the oREV size is determined and the permeability in oREV-scale is calculated.

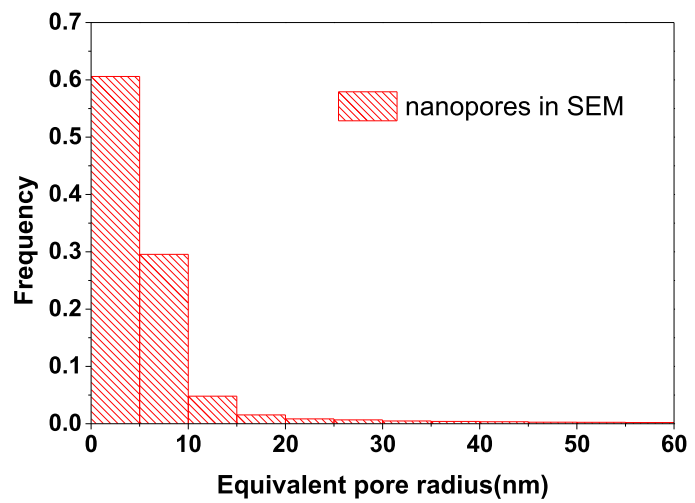

Fig. 11. Comparison of the equivalent pore radius distributions in FIB/SEM and SEM. 


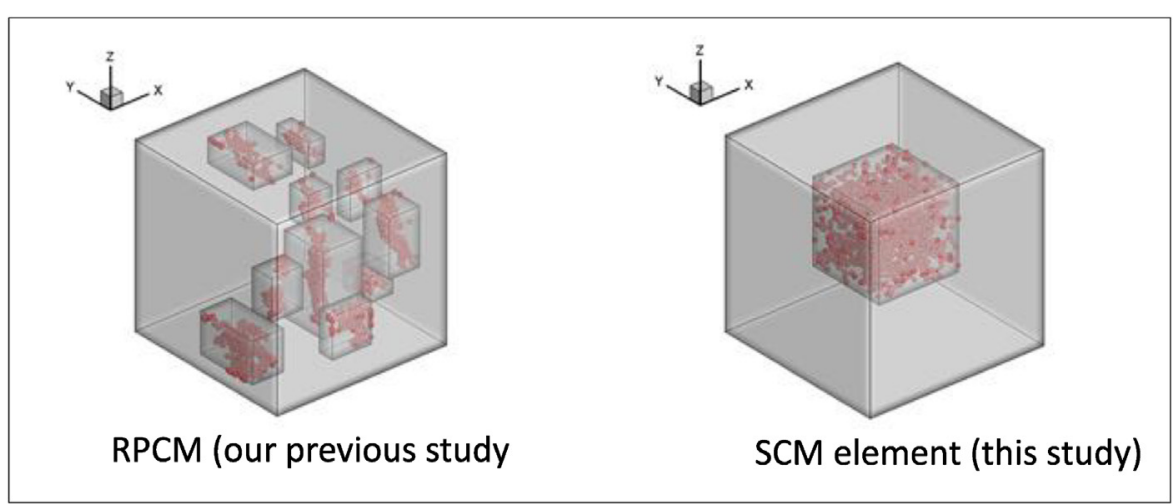

Fig. 12. Models generated from the FIB/SEM images in Fig. 7a.

Table 2

Three calculating methods for the SCM element.

\begin{tabular}{llll}
\hline Methods & OM modeling & IOM modeling & Coupling method \\
\hline Method 1 & PNM & FVM & Mortar \\
Method 2 & Pore cluster equation & FVM & Mortar \\
Method 3 & Series-parallel model as Eq. (6) & \\
\hline
\end{tabular}

Table 3

Characteristic parameters of OM using Eq. (3).

\begin{tabular}{lllllll}
\hline Parameter & $R_{n t}(\mathrm{~nm})$ & $R_{\text {avg }}(\mathrm{nm})$ & $\Phi_{f}$ & $\tau$ & $D_{f}$ & $K_{d, o m}(\mathrm{nD})$ \\
\hline Value & 9.06 & 6.43 & $0.49 \%$ & 3.06 & 2.18 & 8.10 \\
\hline
\end{tabular}

Table 4

Base case input parameters.

\begin{tabular}{ll}
\hline State parameters & Value \\
\hline Average pressure of the inlet and outlet boundaries (MPa) & $0.2-40$ \\
Absolute temperature, T (K) & 311 \\
Gas type & Methane, $16 \mathrm{~g} / \mathrm{mol}$ \\
Diameter of a gas molecule $(\mathrm{nm})$ & 0.38 \\
TMAC & 0.8 \\
\hline
\end{tabular}

\section{Model establishment}

The establishment process of the SCM is presented in this section, as shown in Fig. 2. Multiscale measurements as 3D FIB/SEM and 2D SEM imaging of the same shale sample are adopted in the calculation of shale permeability in oREV-scale. Some assumptions in establishing the model are made as follows:

(1) the type and pore-scale characteristic parameters of the OMs are the same;

(2) the OM content is positive correlation to its volume.

Additionally, the estimation methods of fluid properties are presented in Appendix A.

The algorithm presented in Fig. 2 is as follows:

- On the one hand, the pore-scale characteristic parameters of OM are determined based on 3D FIB/SEM images with the size of $4 \times 4 \times 4 \mu \mathrm{m}^{3}$ and resolution of $4 \mathrm{~nm}$. High-resolution images and simulations in nanopore-scale not only provide improved measurement of fundamental geometry, it also helps advance the understanding of the influence of non-Darcy effect. Instead of assuming an average pore size throughout the system, the SCM element coupled of $\mathrm{OM}$ and IOM is built conditioned to the true pore-radius distribution, throat-radius distribution, coordination number distribution and the content of OM etc.

- In the SCM element, reasonable equivalence is conducted by removing redundant parameters which are not influential to model output such as the spatial distribution of OM/IOM. The permeability of OM is calculated by Jiang's equation (Jiang et al., 2017), and then the permeability of SCM element is calculated using a newly proposed equation based on series-parallel model.

- On the other hand, since the obtained information from the highresolution images is usually highly localized and the nanopore-scale models are computationally limited to small domain size, measurements and simulation in larger scale are involved. A 2D SEM image with the size of $320 \times 320 \mu \mathrm{m}^{2}$ and resolution of $4 \mathrm{~nm}$ is applied to get the OM content distribution to involve the $\sim 100 \mu \mathrm{m}$ magnitude heterogeneity.

- A stochastic SCM with the size of $320 \times 320 \times 320 \mu \mathrm{m}^{3}$ is constructed conditioned to the OM content distribution and then divided into $4 \times 4 \times 4 \mu^{3}$ elements. The pore-scale properties of OM in those elements are assumed to be the same, but a different selected OM content from its distribution is assigned at the randomly selected element.

- A Dirichlet boundary condition is applied to SCM with constant inlet and outlet pressure. And the apparent permeability of the coupled model can be calculated. The oREV size is determined by comparing the permeability of different size of sub-blocks of the SCM.

\subsection{Establishment of the SCM element}

\subsubsection{Introduction of the SCM element}

From FIB/SEM images, the pore-scale parameters of shale are obtained. Then, the SCM element is built based on the statistics information of the characteristic parameters: the pore-radius distribution, throat-radius distribution and coordination number distribution and the content of OM etc., as shown in Fig. 3. In the SCM element, a generation method of random PNM based on the work of Idowu and Blunt (2010) is adopted for the reconstruction of 3D structures of OM. The equivalence changes the dispersed OMs into a composite block. It is also worth mentioning that the sectional porosity of the boundary face of OMs, defined as the ratio of the pore surface to the sectional surface in the boundary face, are averaged into the SCM element to reflect the anisotropy of OM. And the surface fractal dimension of the OMs obtained from FIB/SEM images are also homogenized.

\subsubsection{Permeability calculation for the SCM element}

As to the IOM in the SCM element, the interaction among IOM and microfractures was homogenized as a single continuum medium. Gas flow in IOM is considered to be dominated by Darcy flow and modeled by FVM. The steady conservation equation for FVM is 


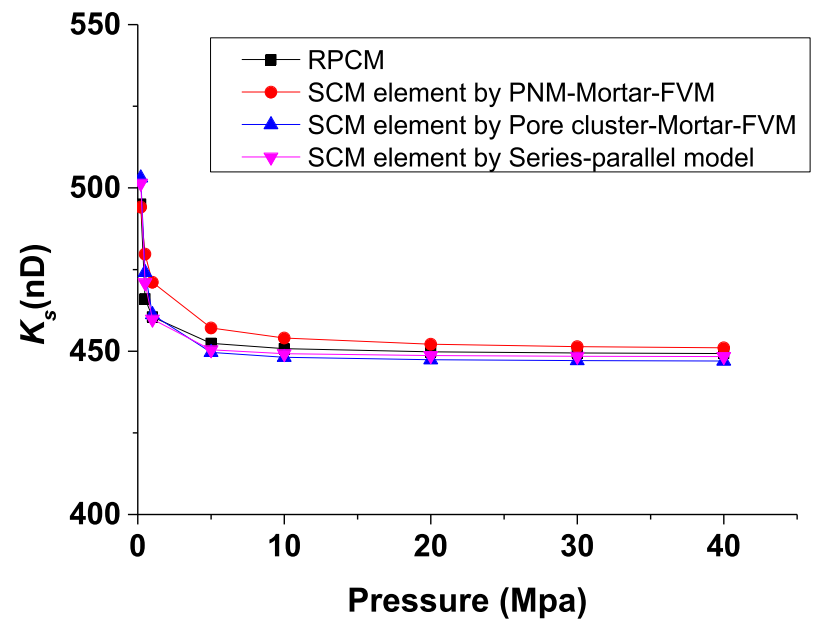

(a)

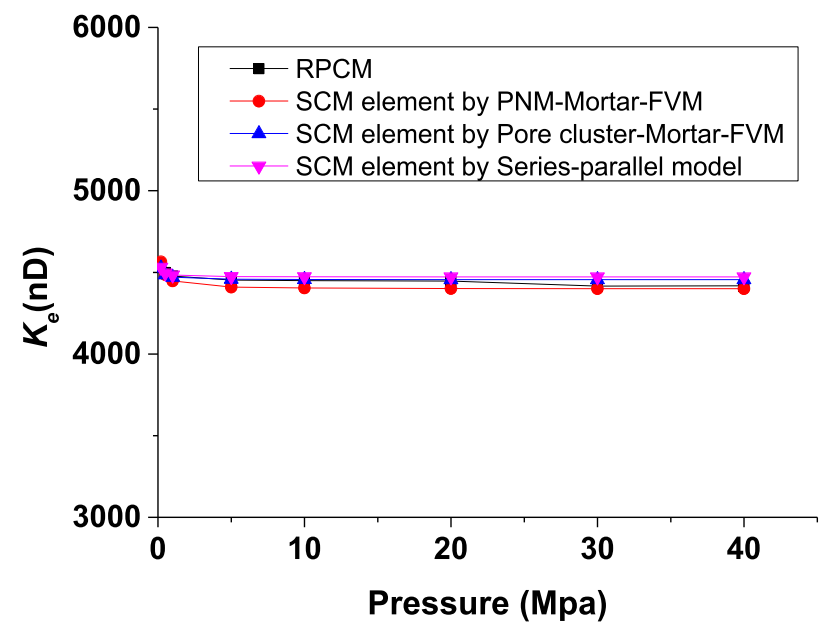

(c)

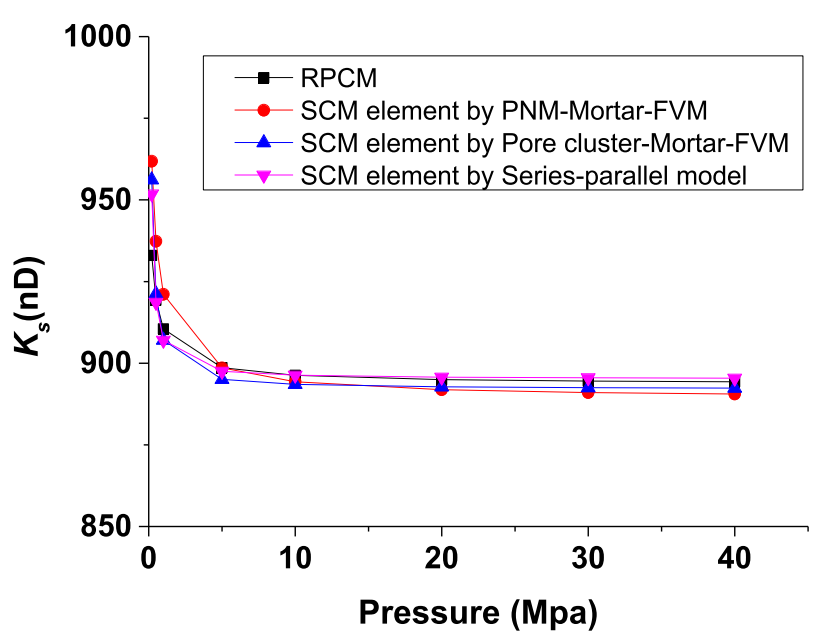

(b)

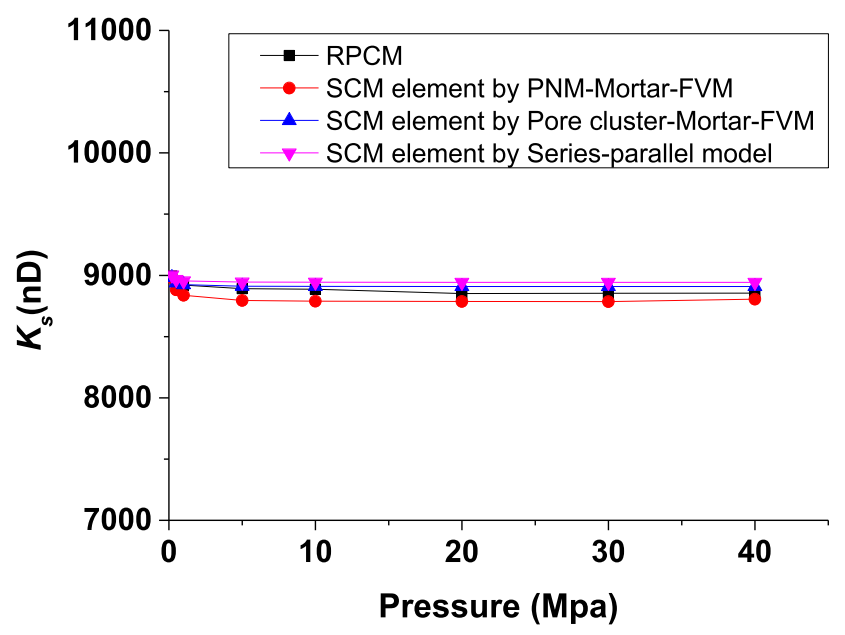

(d)

Fig. 13. $K_{e}$ of the SCM element in the three calculating methods for SCM element at four $K_{i o m}$. (a) $K_{i o m}=500 \mathrm{nD}$, (b) $K_{i o m}=1000 \mathrm{nD}$, (c) $K_{i o m}=5000 \mathrm{nD}$, (d) $K_{\text {iom }}=10000 \mathrm{nD}$. For comparison, the permeability calculated by RPCM is also plotted in the same figure.

Table 5

Computational efficiency of RPCM and SCM element.

\begin{tabular}{|c|c|c|c|c|}
\hline Methods & Mortar grid at per interface & Total number of Mortar nodes & Computing time (s) & $K_{e}(\mathrm{nD})$ \\
\hline RPCM & $1 \times 1$ & 80 & 2063.28 & 894.30 \\
\hline SCM element by PNM-Mortar-FVM & $1 \times 1$ & 8 & 189.31 & 890.56 \\
\hline SCM element by Pore cluster-Mortar-FVM & $1 \times 1$ & 8 & 56.05 & 892.37 \\
\hline SCM element by Series-parallel model & - & - & 5.03 & 895.42 \\
\hline
\end{tabular}

$\nabla\left(\rho \frac{K_{\text {iom }}}{\mu_{g}} \nabla p\right)=0$

As to the OM in the SCM element, the nanopores of OM form complex topological pore networks, PNM is used to describe the gas transport with considering non-Darcy effect. The general approach to PNM is to impose a mass conservation equation at each pore in the network. For compressible steady flows, the conservation equation for pore $i$ is described as $\sum_{j} \frac{K_{i j} \pi r_{i j}^{2}}{\mu_{g} l_{i j}} \frac{p_{\text {avg }} M}{Z R T}\left(p_{i}-p_{j}\right)=0$

where $p_{i}$ and $p_{j}$ are the pressure of pore $i$ and its adjacent pore $j . r_{i j}$ is the radius of the throat which connects pore $i$ and pore $j ; l_{i j}$ is the throat length; $p_{\text {avg }}$ is the average pressure in the throat $p_{\text {avg }}=\left(p_{i}+p_{j}\right) / 2 ; K_{i j}$ is the apparent permeability of the throat considering the viscous flow, slip effect, Knudsen diffusion and pore surface roughness (Darabi et al., 2012; Jiang et al., 2017).

In addition to PNM, Jiang et al. (2017) proposed a simple equation 


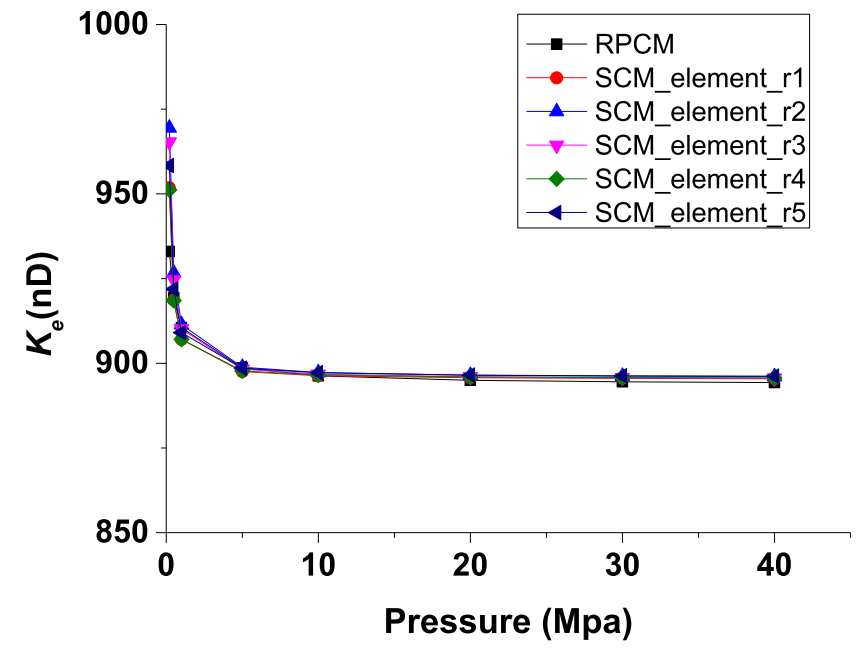

Fig. 14. Permeability of five random realizations of SCM element (SCM_element_r1 r5) with the data in Section 4.2. For comparison, the permeability calculated by RPCM is also plotted in the same figure.

to speed up the permeability computation in the pore networks by determining the characteristic parameters of the pore cluster. The permeability of the pore cluster is defined as:

$\left\{\begin{array}{l}K_{\text {om }}=\frac{2 R_{n t} \mu_{g} \phi_{f}}{3 \rho_{\text {avg }} \tau}\left(\frac{d_{m}}{2 R_{n t}}\right)^{D_{f}-2} \sqrt{\frac{8 M}{\pi R T}}+K_{d, o m}\left(1+\sqrt{\frac{8 \pi R T}{M}} \frac{\mu_{g}}{R_{\text {avg }} P_{\text {avg }}}\left(\frac{2}{\alpha}-1\right)\right) \\ R_{n t}=\left(\frac{1}{N} \sum_{i=1}^{N}\left(\frac{1}{r_{i}}\right)^{D_{f}-3}\right)^{-\frac{1}{D_{f}-3}} \\ R_{\text {avg }}=\left(\frac{1}{N} \sum_{i=1}^{N} \frac{1}{r_{i}}\right)^{-1}\end{array}\right.$

where $\Phi_{f}$ is flowing porosity, defined as the ratio of the void volume with flux magnitudes larger than 0 to the total volume. $d_{m}$ is the diameter of a gas molecule $(0.38 \mathrm{~nm}), r_{i}$ is the radius of throat $i$.

The permeability of the SCM element, $K_{e}$, can be calculated by Mortar coupling of OM and IOM. Mortar is a domain decomposition method convenient for parallel computing, and it is extended to porescale models by Balhoff (Balhoff et al., 2008; Mehmani and Balhoff, 2014). In Cao et al. (2017), Mortar is applied for the first time to connect OM and IOM in shale. In this study, a new equation is proposed to accelerate the computation by series-parallel model according to the water-electric similarity principle. Taking $\mathrm{x}$-direction as an example, the system of $\mathrm{OM}$ and IOM is divided into three part with two cross sections, as shown in Fig. 4.

The permeability of the middle part of the model $K_{e, \text { mid }}$ can be calculated in the parallel of OM and IOM as:
Table 6

Parameters of the computer-generated data.

\begin{tabular}{ll}
\hline State parameters & Value \\
\hline Total volumetric fraction of OM & $6.4 \%$ \\
Mean pore-radius (nm) & 20 \\
Mean throat-radius (nm) & 14 \\
Mean coordination number & 5 \\
Standard deviations & 0.05 \\
Porosity of OM & $10 \%$ \\
\hline
\end{tabular}

$K_{e, \text { mid }}=\psi^{2 / 3} K_{o m}+\left(1-\psi^{2 / 3}\right) K_{\text {iom }}$

And the permeability of the whole model can be calculated in the series of the three parts as:

$K_{e}=\frac{K_{\text {iom }}}{\psi^{1 / 3} K_{\text {iom }} / K_{s, \text { mid }}+\left(1-\psi^{1 / 3}\right)}$

Substituting Eq. (4) into Eq. (5), and then combining it with Eq. (3), one obtains:

$\left\{\begin{array}{l}K_{e}=\left[\frac{1}{\psi^{1 / 3} K_{\text {iom }} /\left(\psi^{2 / 3} K_{o m}+\left(1-\psi^{2 / 3}\right) K_{\text {iom }}\right)+\left(1-\psi^{1 / 3}\right)}\right] K_{\text {iom }} \\ K_{\text {om }}=\frac{2 R_{n t} \mu_{g} \phi_{f}}{3 \rho_{\text {avg }} \tau}\left(\frac{d_{m}}{2 R_{n t}}\right)^{D_{f}-2} \sqrt{\frac{8 M}{\pi R T}}+K_{d, o m}\left(1+\sqrt{\frac{8 \pi R T}{M}} \frac{\mu_{g}}{R_{\text {avg }} p_{\text {avg }}}\left(\frac{2}{\alpha}-1\right)\right)\end{array}\right.$

\subsection{Establishment of SCM}

From 2D SEM images, it is obvious that the heterogeneity still exists out of the visual range of FIB/SEM images, as shown in Fig. 5. In order to reconstruct a model with big enough size for oREV searching, the normalized probability distribution function (PDF) of the OM content, $\psi$ (defined as the volumetric fraction of $\mathrm{OM}$ in an element) is obtained through element division of the SEM image, as shown in Fig. 5. From the analysis of the SEM images, we found that the distribution of OM content $\psi$ is lognormal, and the probability distribution satisfies:

$y=f(\psi \mid \mu, \sigma)=\frac{A}{\sigma \sqrt{2 \pi} \psi} e^{-\frac{(\ln \psi-\ln \mu)^{2}}{2 \sigma^{2}}}$

where $\mu$ and $\sigma$ are the characteristic parameters of the distribution. $\mu$ affects the principal part of the distribution, while $\sigma$ inflects the dispersion degree.

With the PDF of $\psi$, a 3D SCM is generated by assigning a selected $\psi$ at a randomly selected location. The pore-scale characteristic parameters of OM in the SCM are the same with the SCM element in Section 3.1 to take the 3D high-resolution information into consideration. And the SCM element serves as a cell of the SCM. It is worth emphasizing

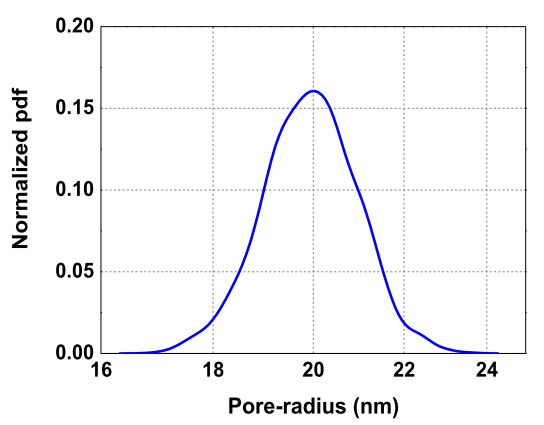

(a)

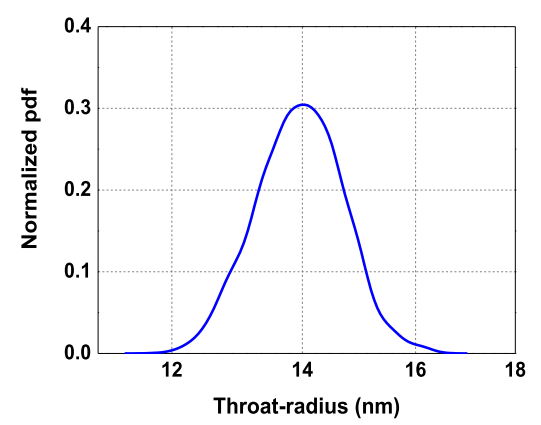

(b)

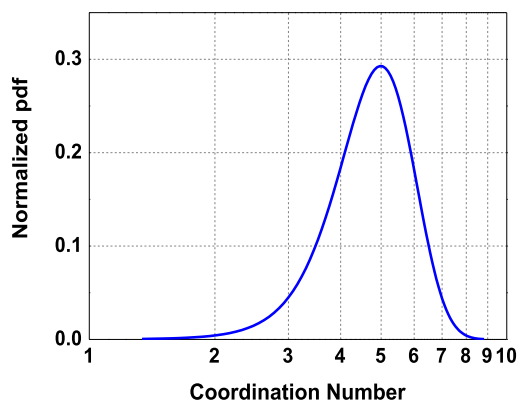

(c)

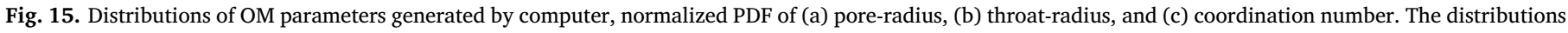
are assumed to be lognormal with fixed mean values and standard deviations. 


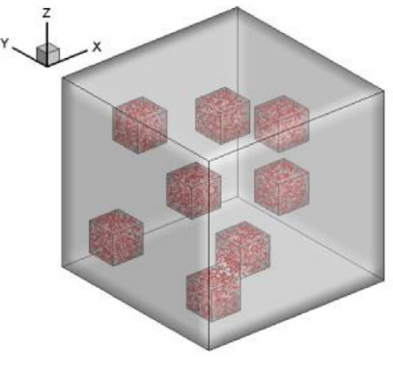

RPCM of the computer-generated data

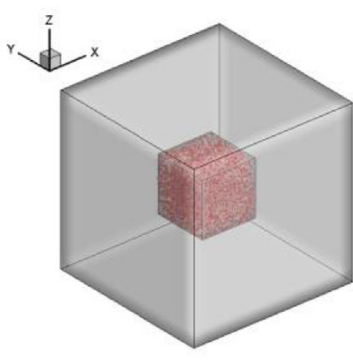

SCM element of the computer-generated data

Fig. 16. Models generated from the computer-generated data.

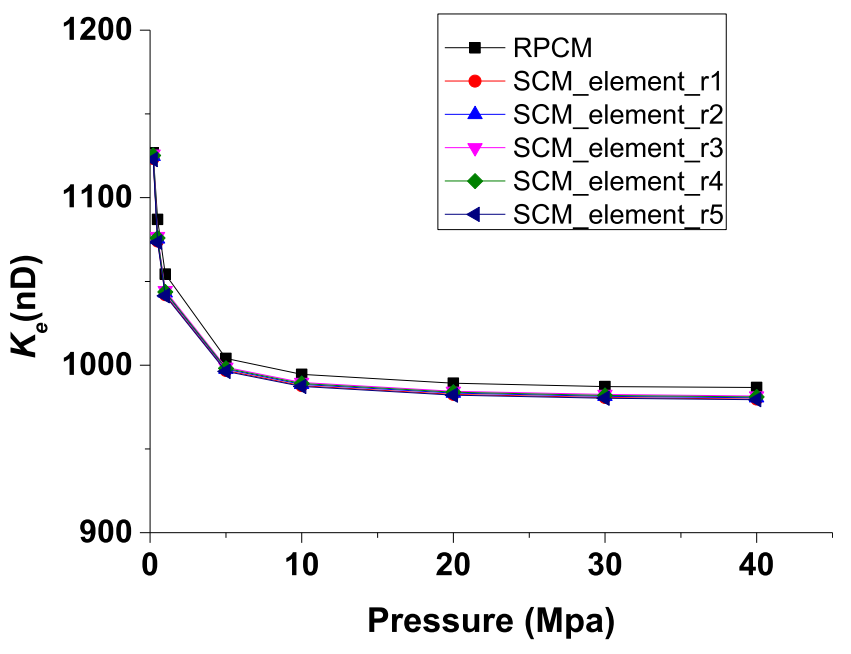

Fig. 17. Permeability of five realizations of SCM element (SCM_element_r1 r5) for the computer-generated data. For comparison, the permeability calculated by RPCM is also plotted in the same figure.

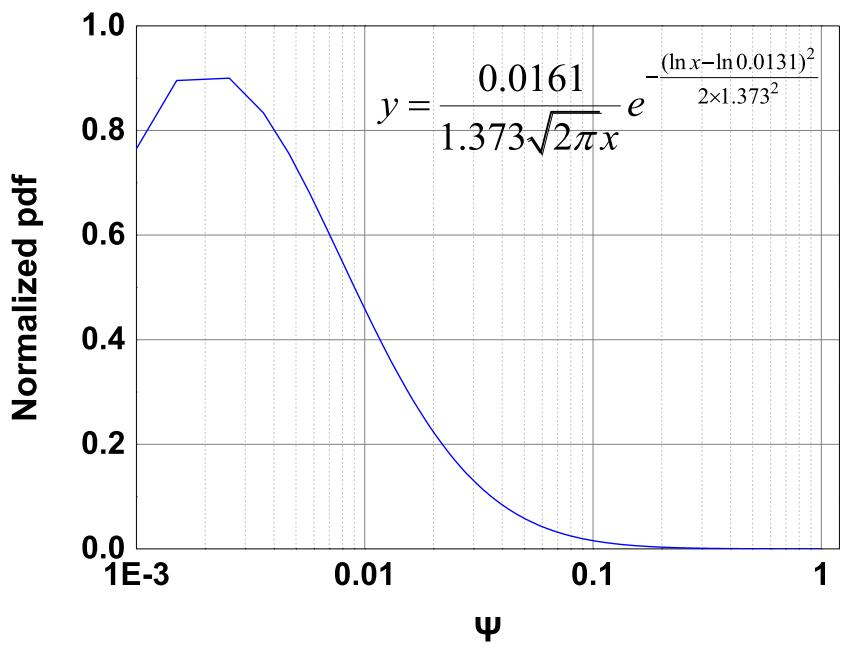

Fig. 18. OM content distribution of the SEM images.

that after the SCM is built, $\psi$ is certain in each SCM element but varied in the whole SCM and subjected to its distribution.

Gas flow in SCM is modeled by FVM. A Dirichlet boundary condition is applied to the coupled model with a constant inlet pressure $p_{\text {in }}$ and outlet pressure $p_{\text {out }}$. And the definition of apparent permeability of SCM, $K_{s}$ is given by:
$K_{s}=\frac{2 Z R T Q_{g} \mu_{g} L_{s}}{A_{s} M\left(p_{\text {in }}^{2}-p_{\text {out }}^{2}\right)}$

where $Q_{g}$ is the average flux at the inlet and outlet, $A_{s}$ is the sectional area of the model, $L_{S}$ is the length along the flow direction.

\subsection{Determination of oREV}

In order to determine the oREV size and its permeability, eight subblocks (Sub1 Sub8) are generated beginning from the vertexes of the SCM, as shown in Fig. 6. Each sub-block has only one element at first and then will be enlarged until the size reaches the boundary. Their permeability is calculated with the same condition. Their permeability will have a big difference when the size of the sub-blocks is small but the relative deviation will approach zero when it comes to oREV-scale. Thus, the oREV size is determined by calculating the permeability in a series of sizes of the sub-blocks. In this study, the relative deviation $\varepsilon$ is defined as the ratio of the standard deviation $\left(\sigma_{K_{S}}\right)$ to the mean value $\left(\bar{K}_{s}\right)$ of the permeability of the sub-blocks, $\varepsilon=\sigma_{K_{s}} / \bar{K}_{s}$. Since that the magnitude of acceptable variation in the measured property and what constitutes a "clear plateau" causes a subjective judgment, a criterion of $\varepsilon<1 \%$ is chose.

\section{Rationality analysis}

In this section, the rationality analysis for the model is presented using a Longmaxi shale sample from the Chongqing Province, China (Sample 1). Firstly, the pore-scale data from FIB/SEM and SEM images are analyzed to verify the assumption that the type and pore-scale characteristic parameters of the OMs are the same. Then, the SCM element is built through an equivalence process. The error caused by the equivalence process in the SCM element and its computational efficiency are analyzed. Finally, the uncertainty caused by the stochastic realization is also investigated. The Longmaxi shale is an organic-rich shale, and is widely developed in the Upper Yangtze region of southern China. The depositional environment of Longmaxi Formation in this study is deep shelf environment. The lithology of the Lower Silurian Longmaxi Formation in the Sichuan Basin, China is mainly the marine organic-rich and quartz-rich siliceous shale. The bottom of the formation is black and grayish-black graptolite rich shale, which is distinct with the lithology on the upper of the formation, dark gray silty shale unequal interbedded with dark gray argillaceous limestone and siltstone laminaes. The TOC of the shale is above $2.0 \%$ at the bottom of the formation (20-50 m thickness). Its vitrinite reflectance (Ro) values range from 1.8 to $4.2 \%$ (Chen et al., 2015a; Liang et al., 2008; Zhang et al., 2018). 


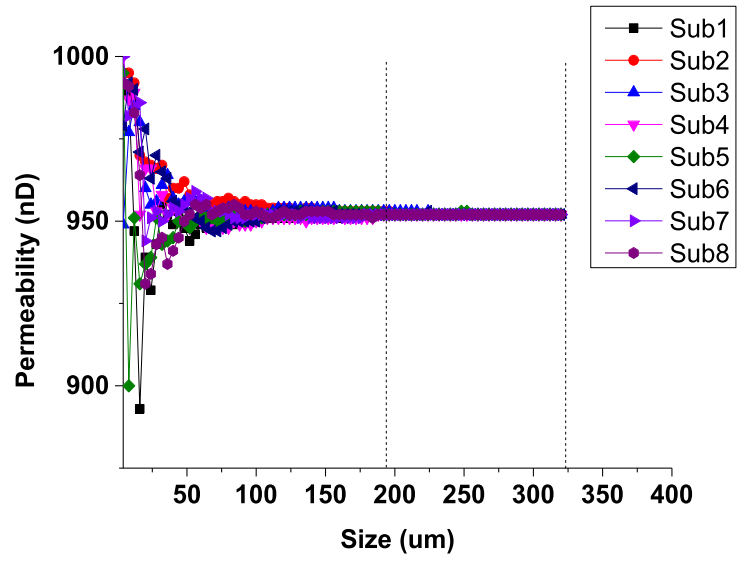

(a)

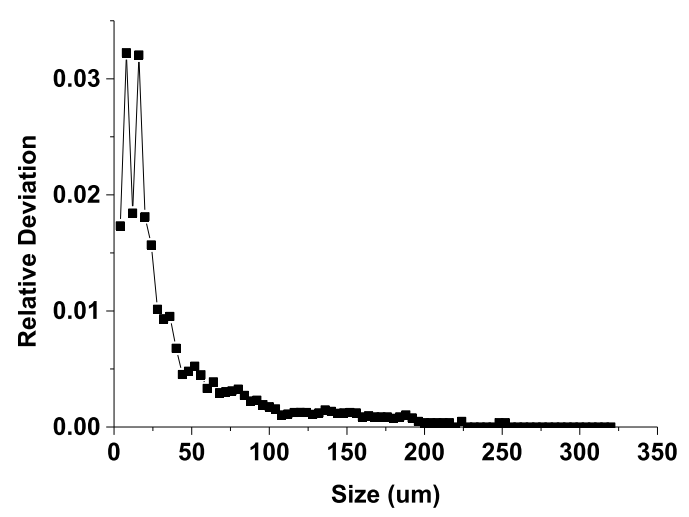

(b)

Fig. 19. Determination of oREV size: (a) the calculation results of permeability of the eight sub-blocks, (b) relative deviation $\varepsilon$.

\subsection{Analysis of organic nanopores}

\subsubsection{Organic nanopores in FIB/SEM}

There are 10 PNMs of OMs extracted from the $4 \mathrm{~nm}$ resolution FIB/ SEM images, as shown in Fig. 7. Fig. 7 demonstrates that the PNMs are isolated from one another and their sizes are different. The parameters of the extracted PNMs are shown in Table 1 . As can be seen, the average pore characteristic parameters such as $C N_{\text {avg }}, R p_{\text {avg }}, R t h_{\text {avg }}, D_{f}$ are close to each other among those OMs while the numbers of pores and throats have a big difference. Results show that the pore properties in those OMs are similar, and the heterogeneity is mainly caused by their sizes. The results suggest that the types and the nanopore properties of the OMs within the several-micrometer size are similar.

The normalized PDFs of the pore-radius, throat-radius and coordination number are obtained by compositing all the PNMs, as shown in Fig. 8. It can be seen that the peak values of the pore-radius, throatradius and coordination number are about $8 \mathrm{~nm}, 4 \mathrm{~nm}$ and 4 . The porescale heterogeneity is strong and should be taken into consideration.

\subsubsection{Organic nanopores in SEM}

In order to analyze the properties of the nanopores within a large domain, the SEM image with the size of $320 \times 320 \mu \mathrm{m}^{2}$ is applied in the section, as shown in Fig. 9a. Using the SEM, twenty OM blocks with the resolution of $4 \mathrm{~nm}$ are obtained and one of them is shown in Fig. $9 \mathrm{~b}$. The image shows that nanopores in $\mathrm{OM}$ are abundant. The statistic results of the equivalent pore radii in the OMs are shown in Fig. 10. Fig. 10 illustrates that the OMs have a similar pore property: the primary parts of the pore radii in different OMs are almost the same, from $3 \mathrm{~nm}$ to $7 \mathrm{~nm}$. The results also suggest that the types of the OMs are basically the same and the pore radius distributions can be considered to be constant within several hundred micrometers.

\subsubsection{Comparison of the organic nanopores in FIB/SEM and SEM}

In this section, the equivalent pore radius distributions of the organic nanopores in FIB/SEM and SEM are compared. Fig. 11 shows that the pore radius in the peak frequency are the same in FIB/SEM and SEM. And the pore radii within $10 \mathrm{~nm}$ accounted for $90 \%$ in both FIB/ SEM and SEM, which indicates that the principal parts of their pore radii are similar. Results also suggest that the FIB/SEM can be considered to be typical in the SEM. We conclude that pore-size distribution of OM obtained from FIB/SEM is representative. Because the poresize distribution is mainly dependent on the type of kerogen which can be the same in the sample. However, the porosity, kerogen volume and permeability of FIB/SEM will not be representative which is mainly caused by the OM/IOM heterogeneity in larger scales. FIB/SEM images are not a suitable oREV for shale. The results is consistent with Kelly et al. (2015). With comprehensive consideration of all the results in Section 4.1, the assumption that the type and pore-scale characteristic parameters of the OMs are the same is reasonable.

\subsection{Deviation analysis of the SCM element}

In this section, the SCM element is built through the integration of OMs, as shown in Fig. 12a. For comparison, a real-physical-coupled model (RPCM) as our previous study (Cao et al., 2017) is also built. RPCM is direct modeling of FIB/SEM images. In RPCM, OM is represented by PNM, IOM is represented by FVM and their interfaces are connected by Mortar. Results in our previous work (Cao et al., 2017) has showed that $\mathrm{OM} / \mathrm{IOM}$ distribution has a negligible influence on shale permeability. For real shale, different blocks of OM can be integrated and thus, the volumetric fraction of OM, pore size distribution and anisotropy etc. can be reflected in the equivalent OM block for a larger scale simulation. In the SCM element, all the OMs are integrated into a single block to get an equivalent result.

In this study, neglecting the OM/IOM distribution within the range of several micrometers, the SCM element is built conditioned to the statistics information of the characteristic parameters: the pore-radius distribution, throat-radius distribution and coordination number distribution and the $\mathrm{OM}$ content etc. And there are three calculating methods for the SCM element, as listed in Table 2. The three methods have different treatment for OM and IOM. In method 1, OM is represented by PNM, IOM is represented by FVM and their interfaces are connected by Mortar. In method 2, PNM is replaced by the pore-cluster equation (Jiang et al., 2017) to simplify the calculation of OM. The critical parameters in the pore-cluster equation are shown in Table 3. In method 3, Mortar method is replaced by series-parallel model as Eq. (6) to simplify the calculation of the whole coupled model for a larger scale simulation. As shale is strongly anisotropic with the presence of bedding, permeability varies in the directions perpendicular and parallel to bedding. In this study we merely calculate the permeability parallel to bedding, a similar simulation can be conducted to other directions.

In order to analyze the error caused by the integration of OMs, the permeability of the SCM element and RPCM is calculated with the characteristic parameters listed in Table 2 and the base case input parameters listed in Table 4 at four $K_{\text {iom }}$ : $500 \mathrm{nd}, 1000 \mathrm{nD}, 5000 \mathrm{nD}$ and $10000 \mathrm{nD}$. And the permeability results are shown in Fig. 13.

From Fig. 13, it can be seen that $K_{e}$ decreases as pressure increases in all those models. It indicates that the non-Darcy effects are captured. When $K_{\text {iom }}=500 \mathrm{nD}$ or $1000 \mathrm{nD}$, the deviation of those models tends to be steady by approximately $1 \%$ for pressure greater than $5 \mathrm{Mpa}$, as gas 


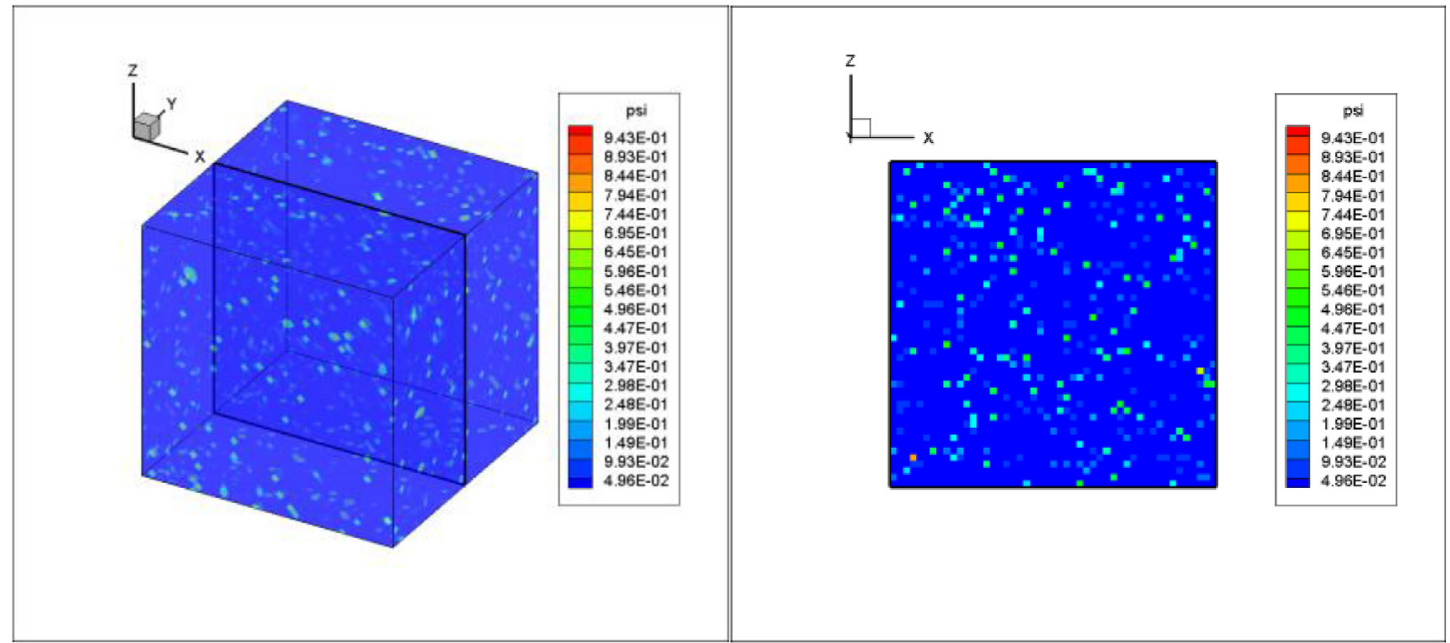

(a)

(b)

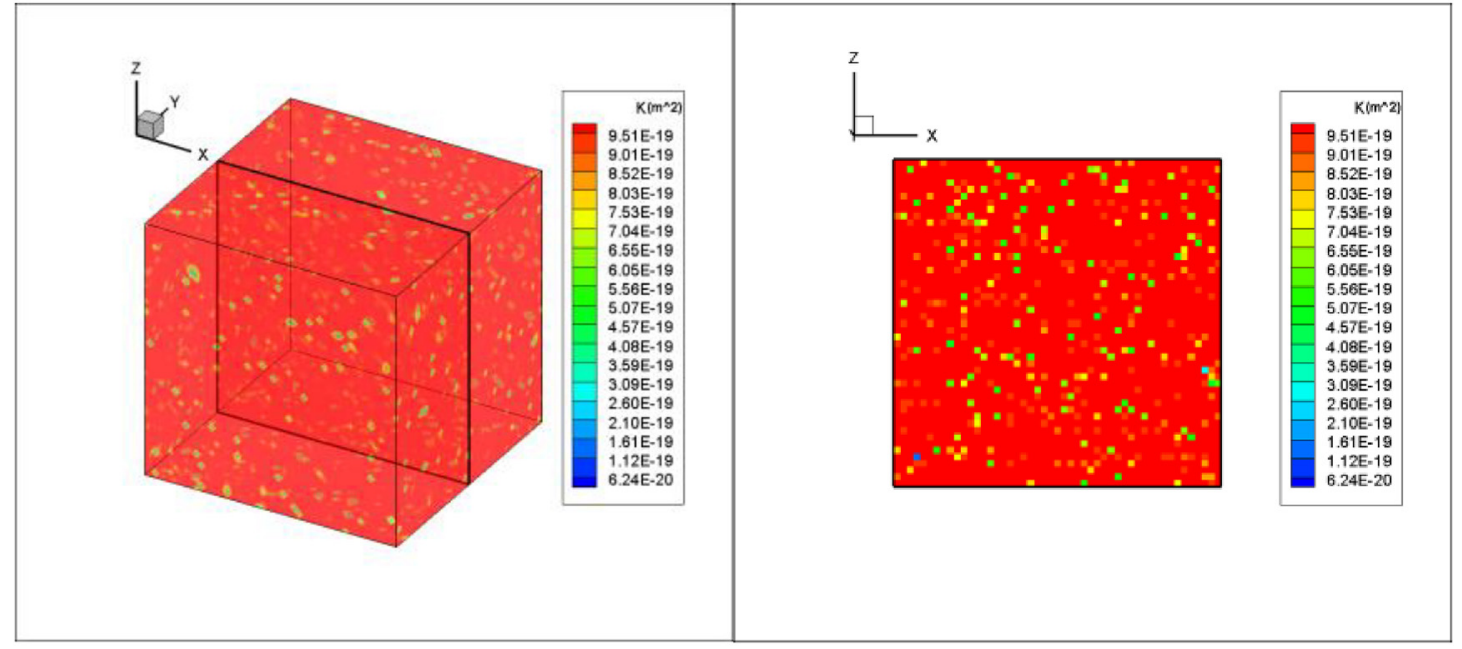

(c)

(d)

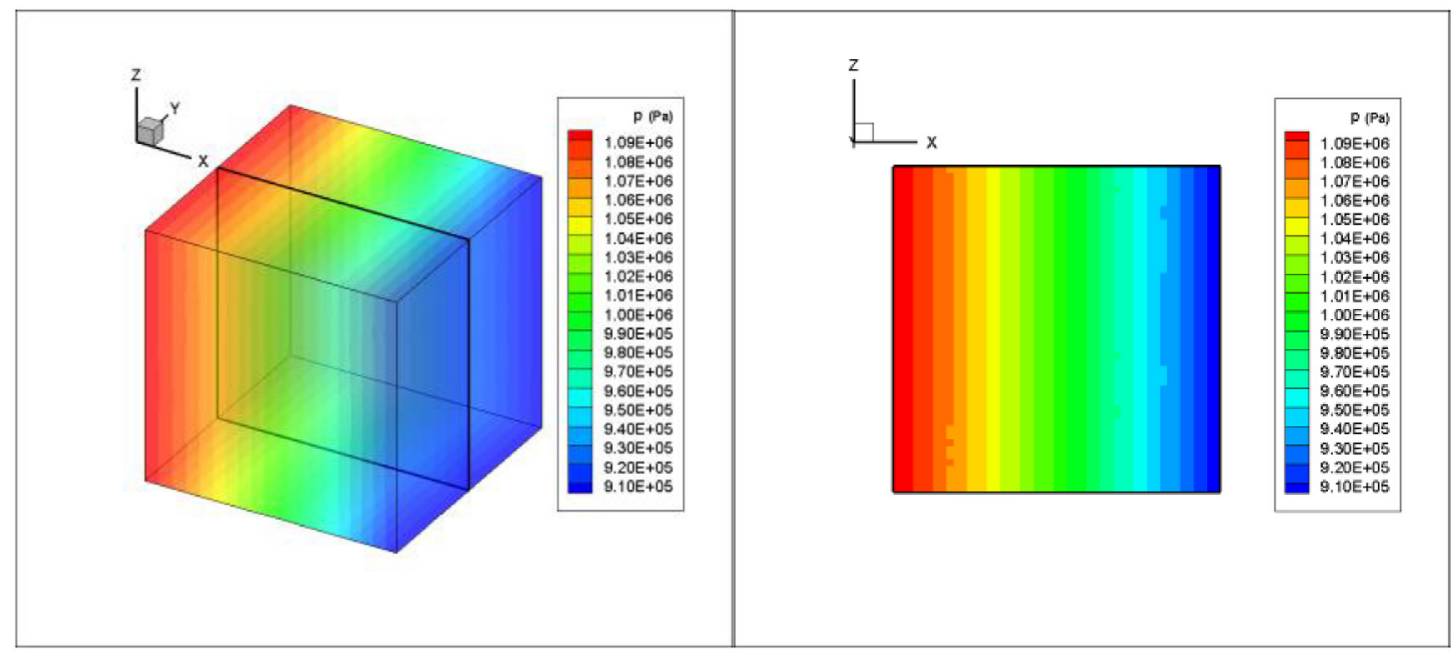

(e)

(f)

Fig. 20. Maps of the calculated parameters in oREV-scale: (a) 3D maps of $\psi$, (c) 3D maps of $K_{s}$, (e) 3D maps of pressure; the 2D maps of the corresponding parameters of the center section in y-direction are also shown for clarity in (b), (d) and (f). 


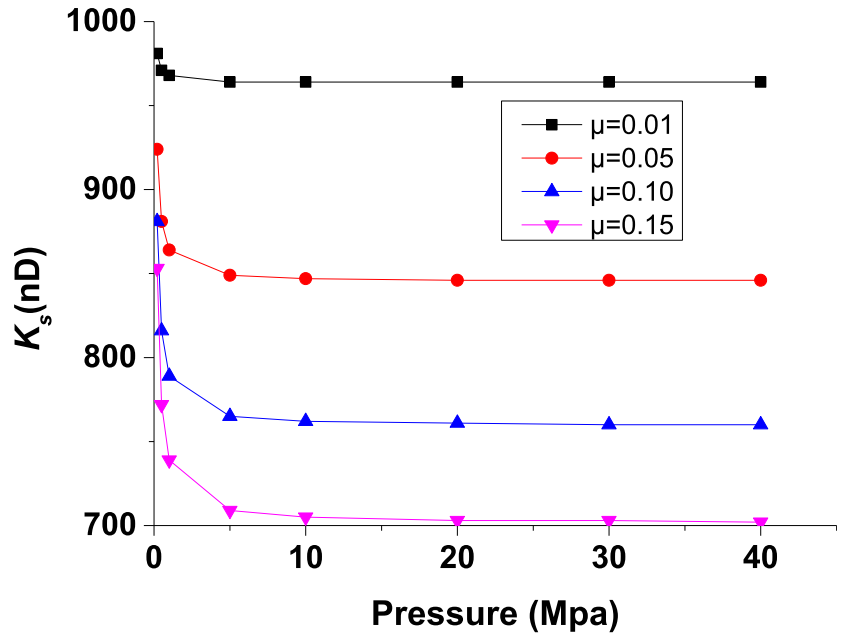

Fig. 21. Sensitivity of $K_{s}$ to $\mu$.

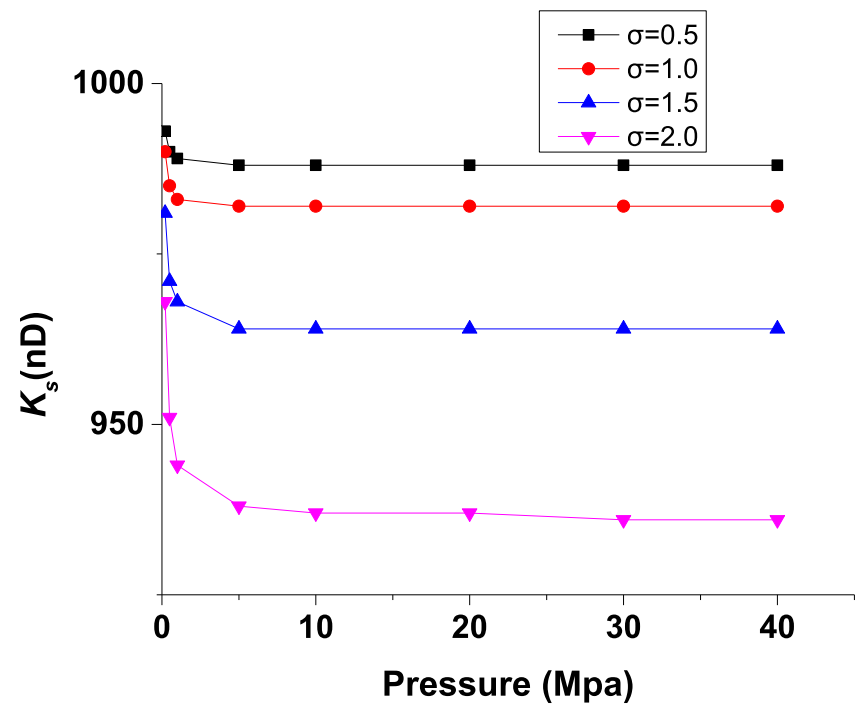

Fig. 22. Sensitivity of $K_{s}$ to $\sigma$.

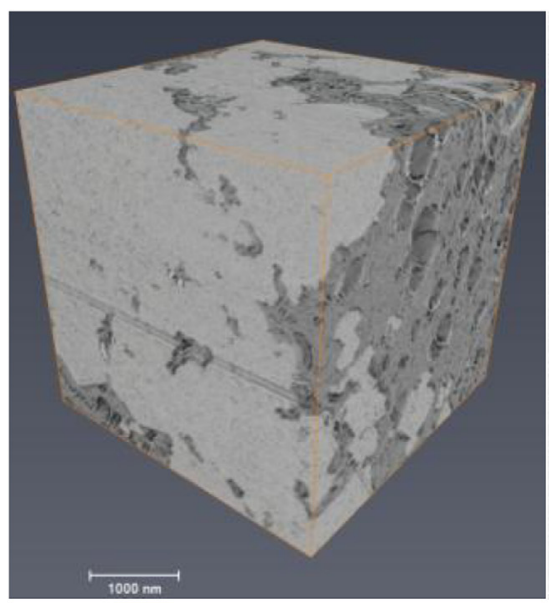

(a) flow tends to be Darcy's flow, and the decrease in pressure increases the deviation with a maximum error of $3 \%$ in $0.2 \mathrm{Mpa}$. When $K_{\text {iom }}=5000 \mathrm{nD}$ or $10000 \mathrm{nD}$, the deviations between those models are within $2 \%$ in a pressure range from $0.2 \mathrm{MPa}$ to $40 \mathrm{MPa}$, because $K_{\text {iom }}$ is much larger than $K_{o m}$ at such conditions and the non-Darcy effects occurring in OM have a small influence on $K_{e}$. In general, the results show that the $K_{e}$ curves of both the coupled models based on physical images (the RPCM) and the statistical information (the SCM element) are close to each other with the permeability deviation $<3 \%$. The results suggest that although stochasticity is introduced by statistics method, the error of SCM element is acceptable. Results also suggest that the spatial distributions of pores and throats also have a negligible influence on $K_{e}$.

Additionally, the computational efficiency of SCM element is analyzed. The computing times of the different models are presented with $K_{\text {iom }}$ of $1000 \mathrm{nD}$ and average pressure of $40 \mathrm{MPa}$, as shown in Table 5. In general, the SCM element using different calculating methods all show a much faster computing than that of RPCM. When both the SCM element and RPCM are calculated using Mortar coupling, the SCM element has a faster computing with a much smaller total number of Motar nodes. And when the SCM element is calculated using series-parallel model, the calculation is extremely simple by Eq. (6). Results show that the efficiency of SCM element using series-parallel model is approximately 400 times that of RPCM. The equivalent model shows a good performance on permeability calculation with a significantly fast computing and an acceptable deviation. For a larger scale simulation, calculating the permeability of shale with several-micrometer size using the SCM element as Eq. (6) is reasonable and high-efficiency.

\subsection{Uncertainty analysis}

As the SCM element have some randomness in the reconstruction of the integrated OM block where the spatial distributions of pores and throats are stochastic. In this section, several random realizations of the same pore-scale data are built to analyze the uncertainty introduced by the equivalence of SCM element. The real shale data in Section 4.2 are used to generate several random realizations, and the permeability will be calculated directly by the series-parallel model. Additionally, in order to assess the uncertainty of SCM element in stochastic data bases, an artificial case is also built with the computer-generated PDFs of poreradius, throat-radius and coordination number. The input parameters for permeability calculation are listed in Table 4 and the $K_{\text {iom }}$ is $1000 \mathrm{nD}$.

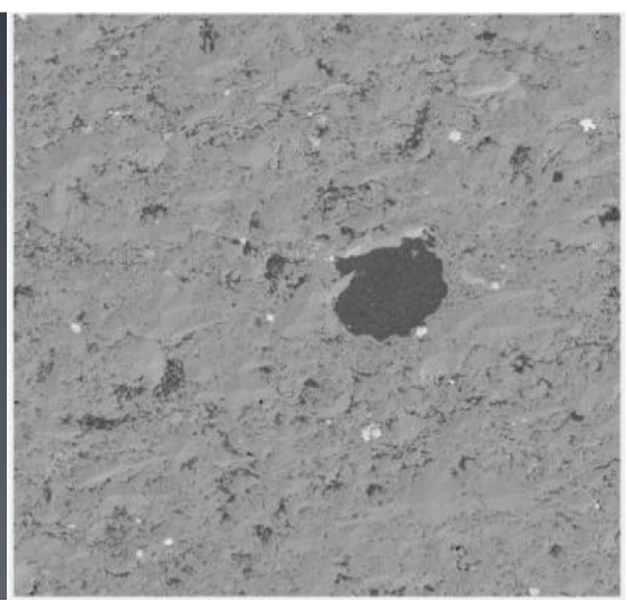

(b)

Fig. 23. Another shale sample (Sample 2) from a different district with the sample in Section 4 (Sample 1). (a) FIB/SEM images, (b) SEM image. 


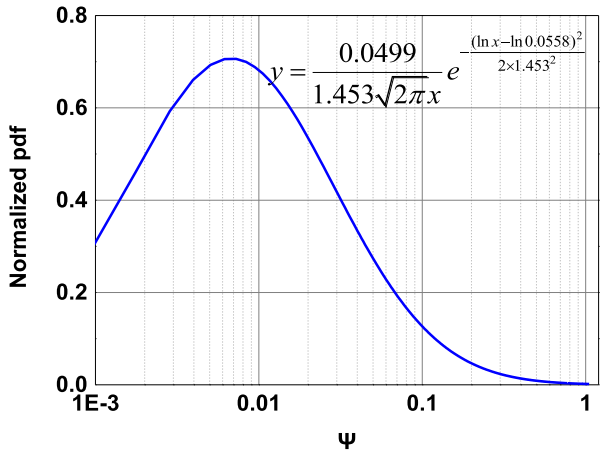

(a)

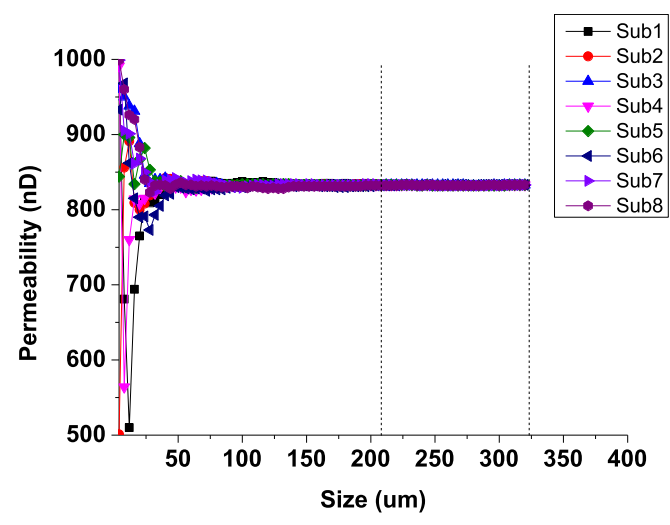

(b)

Fig. 24. (a) OM content distribution of the SEM images in Fig. 23, (b) determination of oREV size: the calculation results of permeability of the eight sub-blocks.

Table 7

Parameters comparison of the two shale samples.

\begin{tabular}{llll}
\hline Parameters & & Sample 1 & Sample 2 \\
\hline Characteristic & $R_{n t}(\mathrm{~nm})$ & 9.06 & 11.89 \\
parameters from & $R_{\text {avg }}(\mathrm{nm})$ & 6.43 & 5.87 \\
3D FIB/SEM & $\Phi_{f}$ & $0.49 \%$ & $0.28 \%$ \\
images & $\tau$ & 3.06 & 1.75 \\
& $D_{f}$ & 2.18 & 2.20 \\
& $K_{d, o m}(\mathrm{nD})$ & 8.10 & 19.33 \\
\hline $\begin{array}{c}\text { Characteristic } \\
\text { parameters from } \\
\text { 2D SEM image }\end{array}$ & $\mu$ & 0.013 & 0.056 \\
\hline $\begin{array}{c}\text { Parameters in oREV- } \\
\text { scale }\end{array}$ & oREV size & $192 \times 192 \times 192$ & $208 \times 208 \times 208$ \\
& $\left(\mu \mathrm{m}^{3}\right)$ & & \\
& Darcy & 952 & 833 \\
& permeability & & \\
\hline
\end{tabular}

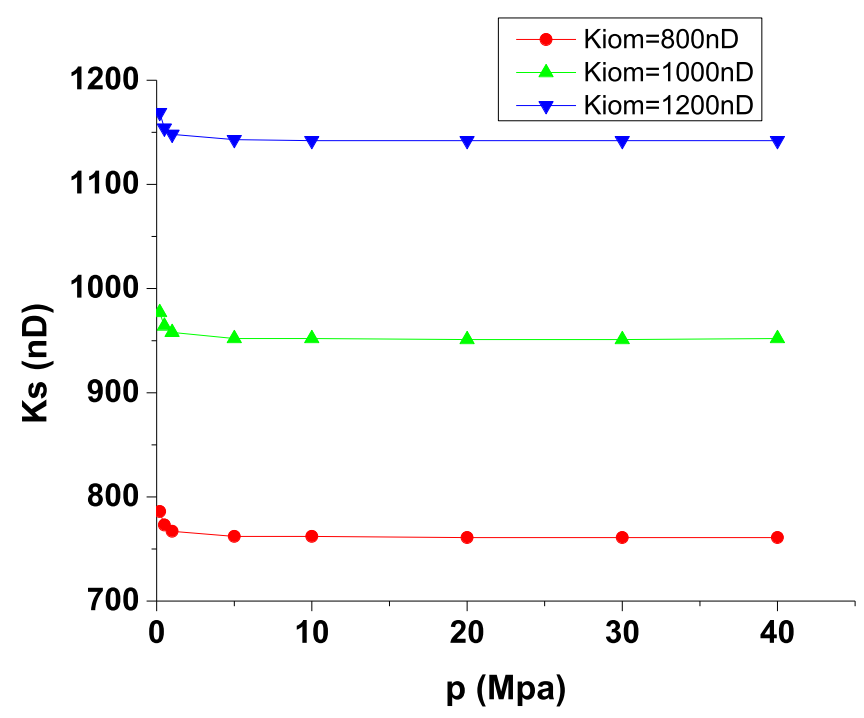

Fig. 25. Sensitivity of $K_{s}$ to $K_{\text {iom }}$.

\subsubsection{Real-Shale data}

The real shale data in Section 4.2 are used to generate five realizations of SCM element and the calculated $K_{e}$ is shown in Fig. 14. Fig. 14 demonstrates that when pressure is $0.2 \mathrm{MPa}$, the error caused by the equivalence is $4 \%$; while the pressure is higher than $0.5 \mathrm{MPa}$, the error is within $1 \%$. The results show that though stochasticity is introduced among the equivalent process, permeability calculation does not suffer much from uncertainty for the real shale data.

\subsubsection{Computer-generated data}

In this section, the PDFs of pore-radius, throat-radius and coordination number of OM are assumed to be lognormal to extend the application of our model to a stochastic case, as showed in Fig. 15. With the parameters listed in Table 6, the RPCM and SCM element are generated, as shown in Fig. 16. In order to analyze the uncertainty, five random realizations of SCM element are generated and their permeability values are calculated, the results are shown in Fig. 17.

Fig. 17 demonstrates that the error between the RPCM and the five realizations of SCM element is less than $2 \%$. The results suggest that permeability calculation also does not suffer much from uncertainty for a random data.

\section{Results and discussions}

\section{1. oREV construction}

Through element division of the SEM image in Fig. 9a of Sample 1, the OM content distribution is obtained, as shown in Fig. 18.

From the analysis of the OM content distribution, we find that the distribution of OM content, $\psi$ is lognormal, and its probability satisfies:

$y=\frac{0.0161}{1.373 \sqrt{2 \pi} \psi} e^{-\frac{(\ln \psi-\ln 0.0131)^{2}}{2 \times 1.373^{2}}}$

Using the OM content distribution, a 3D SCM is built by assigning a selected $\psi$ at a randomly selected location. And its pore-scale characteristic parameters of OM are the same as the SCM element, as listed in Table 3. In order to determine the oREV size of the SCM, eight subblocks (Sub1 $\sim$ Sub8) are generated beginning from the vertexes of the SCM. The base case input data are the same as Table 4, and the Darcy's flow is assumed here with the $K_{\text {iom }}$ of $1000 \mathrm{nD}$. The calculation results of $K_{s}$ of the eight sub-blocks and the relative deviation are presented in Fig. 19. $K_{s}$ fluctuates in the small sample size, and then reaches a plateau of $952 \mathrm{nD}$ around sample size of $192 \times 192 \times 192 \mu \mathrm{m}^{3}$ and the relative deviation between the eight sub-blocks goes approximately to zero $(<1 \%)$, meaning that the oREV size could be $192 \times 192 \times 192 \mu \mathrm{m}^{3}$.

In the oREV-scale, different sub-blocks have the same permeability, and the permeability will still be constant in a relatively lager domain. However, a model with the size smaller than oREV will induce large errors. There is a trade-off between accuracy and efficiency, regarding to them simultaneously, simulating in the oREV-scale is the optimal way. We present the maps of $\psi, K_{s}$, pressure of a realization of SCM in 


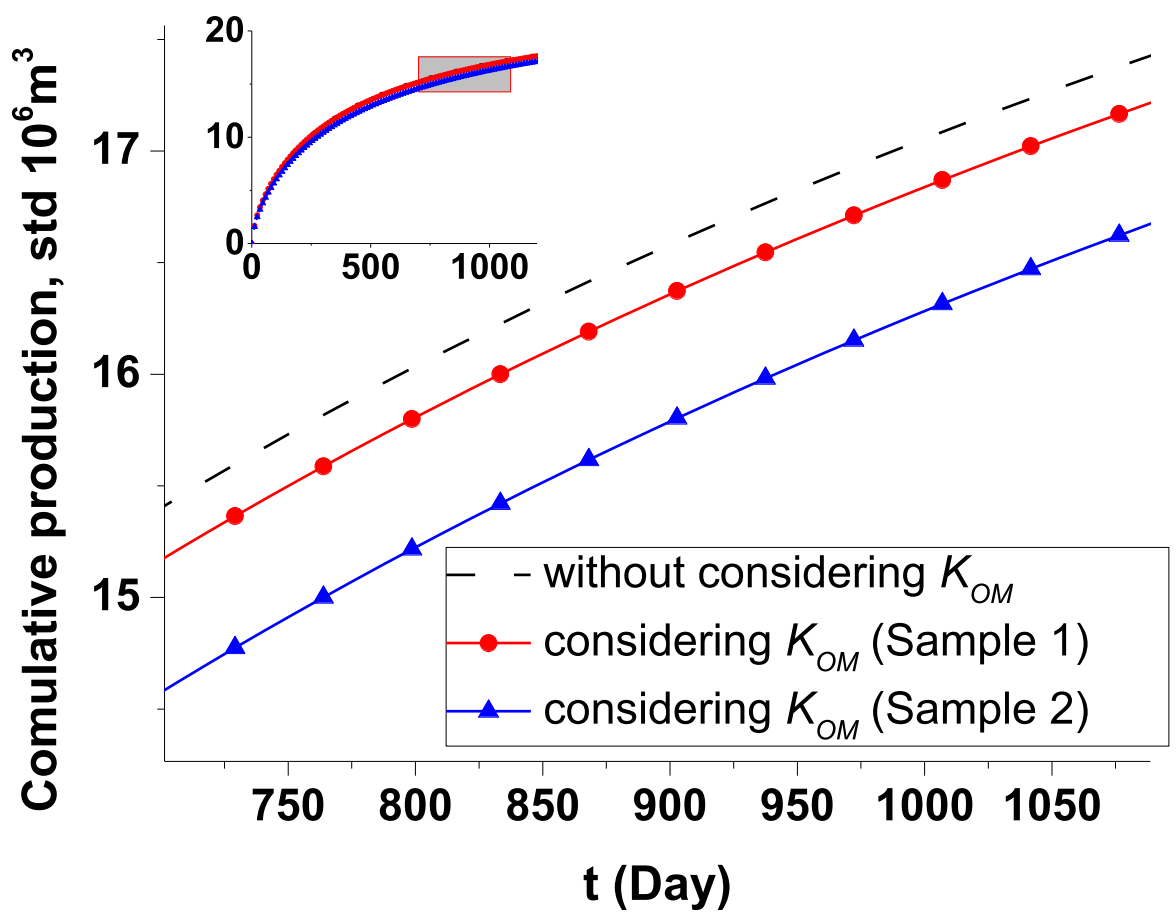

Fig. 26. Difference between considering/without considering $K_{\text {om }}$.

oREV-scale, as shown in Fig. 20. Results show that (1) the heterogeneity within the oREV-scale model is strong so a smaller model will be unsuitable, (2) a higher $\psi$ induces a lower $K_{s}$, as the permeability of OM is lower than that of IOM, and (3) the pressure contours bend more seriously as $\psi$ increases in a local place as the pressure-drop is big in a lowpermeability area.

\subsection{Sensitivity analyses in oREV-scale}

In this section, the sensitivity analyses of the oREV-scale permeability to the OM content distribution, shale sample and $K_{\text {iom }}$ are conducted.

\subsubsection{Sensitivity of $K_{s}$ to the $O M$ content distribution}

The sensitivities of $K_{s}$ to the OM content distribution are performed in oREV-scale using Sample 1. The value of pressure varies between $0.2 \mathrm{MPa}$ and $40 \mathrm{MPa}$. Assuming a constant $\sigma$ of 1.5, the sensitivity of $K_{s}$ to $\mu$ is presented in Fig. 21. Results show that the value of $K_{s}$ decreases as $\mu$ increases. The result can be explained as follows: a larger $\mu$ induces a higher total OM content, because OM has a lower permeability than that of IOM, permeability of the coupled system decreases as the total OM content increases. Assuming a constant $\mu$ of 0.01 , the sensitivity of $K_{s}$ to $\sigma$ is presented in Fig. 22. Results show that the value of $K_{s}$ decreases as $\sigma$ increases. $\sigma$ inflects the dispersion degree of OM content, and the heterogeneity of the coupled system increases as $\sigma$ increases. More low-permeability parts and high-permeability parts will be induced with increasing $\sigma$. Through the series-parallel analysis, the total permeability will decreases as the low permeability parts increase. Results suggest that the permeability of shale decreases as the heterogeneity of OM size increase.

\subsubsection{Sensitivity of $K_{s}$ to the district of shale sample}

Another shale sample from Lower Silurian Longmaxi formations in the Qiliao Section, Eastern Chongqing is employed to analyze the sensitivity of $K_{s}$ to the district of shale sample, as shown in Fig. 23. The OM content distribution and the determination of oREV are presented in Fig. 24. With comparison to the sample in Section 4, the characteristic parameters of the two shale sample are listed in Table 7. The Darcy permeability of OM in Sample 2 is about double that of Sample 1, shows a higher conductivity. The value of $\mu$ in Sample 2 is about 5 times that of Sample 1 which shows a higher total OM content. As the permeability of OM is much lower than that of IOM, the permeability in oREV-scale decreases as the total OM content increases. Though their oREV sizes are similar, with a value of about $200 \times 200 \times 200 \mu \mathrm{m}^{3}$, their Darcy permeability has much difference with the value of $952 \mathrm{nD}$ and $833 \mathrm{nD}$.

\subsubsection{Sensitivity of $K_{s}$ to $K_{\text {iom }}$}

The sensitivity of $K_{s}$ to $K_{\text {iom }}$ is also investigated using Sample 1. Fig. 25 illustrates the $K_{s}$ curves in different $K_{\text {iom }}$ with pressure varying from 0.2 MPa to $40 \mathrm{MPa}$. A few observations can be made from Fig. 25. First, the increase in pressure decreases the $K_{s}$ in oREV-scale. And $K_{s}$ increases as $K_{\text {iom }}$ increases. Second, $K_{s}$ is not linear superposition or volumetrically weighted average of $K_{\text {om }}$ and $K_{\text {iom }}$. As OM is isolated from one another in the shale matrix, applying area- or volumeweighted averaging method to calculate $K_{s}$ will induce errors.

\subsection{Influence of microscale properties on macroscale behavior}

Using the permeability in oREV-scale, the gas production can be calculated using the simulator presented in Appendix B. The wellknown diffusivity equation is selected, given as:

$\nabla\left(\rho \frac{K_{s}}{\mu_{g}} \nabla p\right)=\frac{\partial\left(\rho \phi_{s}\right)}{\partial t}+\frac{\partial\left(\rho_{s} \rho_{s t p} V_{a b s, s t p}\right)}{\partial t}$

Unlike a traditional way, $K_{s}$ is calculated by the SCM with detailed thinking about the influence of OM. $K_{s}$ changes with pressure and space, so it is dynamic value in the gas production process. Gas productions with and without considering $K_{o m}$ in the multi-stage fractured horizontal well are calculated, as shown in Fig. 26. When $t=1000$ day, (1) for sample 1 , the cumulative production will be underestimated by 0.2 million $\mathrm{m}^{3}$ without consideration of $K_{\text {om }}$; (2) for sample 2 , the cumulative production will be underestimated by 1 million $\mathrm{m}^{3}$. Results show that the macroscale production will be influenced by the microscale properties. Since OMs are dispersed in IOM, if the difference between the permeability of OM and IOM is not taken into consideration 
will induce errors in the gas production. And the impact degree depends on the OM content and its pore abundance and topological structure. Chen (2016) considered that OM has nothing to do with the transport channel and gas flow only occurs in IOM. It's shown that their assumptions hold under certain conditions. Our model can reflect the microscale difference between the shale samples in the macroscale behavior. The parameters of oREV can be used as a new way to evaluate the properties of shale and help find the "sweet spot" and the high quality reservoir. Meanwhile, the model establishes a relationship between microscale and macroscale, which affords a foundation for investigating the multiscale problem in shale.

\section{Conclusion}

This study proposed a statistical-coupled model of OM and IOM for shale permeability calculation in oREV-scale. High-resolution 3D FIB/ SEM images of $4 \times 4 \times 4 \mu \mathrm{m}^{3}$ are employed to determine the characteristic parameters in $\mathrm{OM}\left(R_{n t}, R_{\text {avg }}, \Phi_{f}, \tau, D_{f}, K_{d, o m}\right)$. And then, a larger SEM image is employed to build a $320 \times 320 \mu \mathrm{m}^{2}$ coupled model conditioned to the OM content distribution with the characteristic parameters $(\mu$ and $\sigma$ ) to find the oREV size. The permeability of oREVscale shale is thus calculated and then its sensitivities are also analyzed. Following are the conclusions drawn from the study:

- The average pore characteristic parameters such as $C N_{\text {avg }}, R p_{\text {avg, }}$, $R t h_{a v g}$ and $D_{f}$ are close to each other among those OMs while the numbers of pores and throats have a big difference caused by the sizes of OMs.

- Results show that the $K_{e}$ curves of both the coupled models based on physical images (the RPCM) and the statistical information (the SCM element) are close to each other with the permeability deviation $<3 \%$. But the efficiency of SCM element using seriesparallel model is approximately 400 times that of RPCM. The SCM element shows a good performance on permeability calculation with a significantly fast computing speed and an acceptable deviation in the several-micrometer scale.

- The oREV size of a Longmaxi shale sample from the Chongqing Province, China is about $200 \times 200 \times 200 \mu \mathrm{m}^{3}$.

- We performed sensitivity analyses of $K_{s}$ to various parameters. The model results show that an increase in $\mu, \sigma$ or average pressure will decrease $K_{s}$. These results also suggest that permeability estimation in shale systems solely based on average OM block size could be erroneous as the permeability is sensitive to its content distribution. $K_{s}$ will also has a big difference in different shale sample. The increase in $K_{\text {iom }}$ increases $K_{s}$, and $K_{s}$ is not linear superposition or volumetrically weighted average of $K_{\text {om }}$ and $K_{\text {iom }}$.

The new model can establish a relationship between microscale properties and macroscale behavior, with deeply taking the influence of OM into consideration.

\section{Acknowledgments}

This work is supported by the National Natural Science Foundation of China (Grant No. 41690132), the Major National Science and Technology Special Program of China (Grant No. 2017ZX05037-001), the Strategic Priority Research Program of the Chinese Academy of Sciences (grant No. XDA14010101), the National Natural Science Foundation of China (Grant No. 41574129) and the Strategic Priority Research Program of the Chinese Academy of Sciences (Grant No. XDB10020302).

\section{Appendix A. Estimation of fluid properties}

The gas compressibility factor $Z$ can be calculated using pseudo reduced pressure $\left(p_{p r}\right)$ and pseudo reduced temperature ( $\left(T_{p r}\right)$ based on the following equations (Mahmoud, 2013).

$p_{p r}=p / p_{c}$

$T_{p r}=T / T_{c}$

$Z=\left(0.702 e^{-2.5 T_{p r}}\right) p_{p r}^{2}-\left(5.524 e^{-2.5 T_{p r}}\right) p_{p r}+0.044 T_{p r}^{2}-0.164 T_{p r}+1.15$

where $p_{c}$ and $T_{c}$ are the critical pressure and temperature, respectively.

Viscosity $\mu_{g}$ is obtained using the method proposed by Lee et al. (1966).

$\mu_{g}=\left(1 \times 10^{-4}\right) A \exp \left(B \rho^{C}\right)$

$A=\frac{(9.379+0.01607 M)(1.8 T)^{1.5}}{209.2+19.26 M+1.8 T}$

$B=3.448+0.01009 M+986.4 /(1.8 T)$

$C=2.447-0.2224 B$

where $\rho$ is the gas mass density in $\mathrm{g} / \mathrm{cm}^{3}, T$ is the temperature in Kelvin, $M$ is the molar mass in $\mathrm{g} / \mathrm{mol}$, and $\mu_{\mathrm{g}}$ is in mPa.s.

\section{Appendix B. Macroscale behavior simulator}

Eq. (10) is discretized by FVM based on PEBI grid and solved by the implicit algorithm. The amount of adsorption is modeled by the Langmuir isotherm equation:

$V_{a b s, s t p}=\frac{V_{L} p}{p_{L}+p}$

$V_{L}$ and $p_{L}$ are set to be $0.01 \mathrm{~m}^{3} / \mathrm{kg}$ and 7.5 MPa, respectively (Civan et al., 2010). $\rho_{\text {stp }}$ is the gas density at standard temperature and pressure, STP (set to be $0.714 \mathrm{~kg} / \mathrm{m}^{3}$ ). We assume a multi-stage hydraulic fractured horizontal well at a constant pressure of $0.3 \mathrm{MPa}$ in a homogeneous, isotropic, isothermal, closed acting reservoir at initial pressure $55 \mathrm{MPa}$. The reservoir size is $350 \mathrm{~m} \times 200 \mathrm{~m}$. The half-length, spacing and aperture of the fractures are $60 \mathrm{~m}, 50 \mathrm{~m}$ and $0.0015 \mathrm{~m}$ (Singh and Cai, 2018), the simulation domain and the PEBI grids are shown in Fig. B1. Gas reservoir temperature is $311 \mathrm{~K} . \Phi_{s}$ is $0.05 . K_{\text {iom }}$ is set to be $1000 \mathrm{nD}$. The multi-stage hydraulic fracturing reservoir and its grids are shown in Fig. B1. 

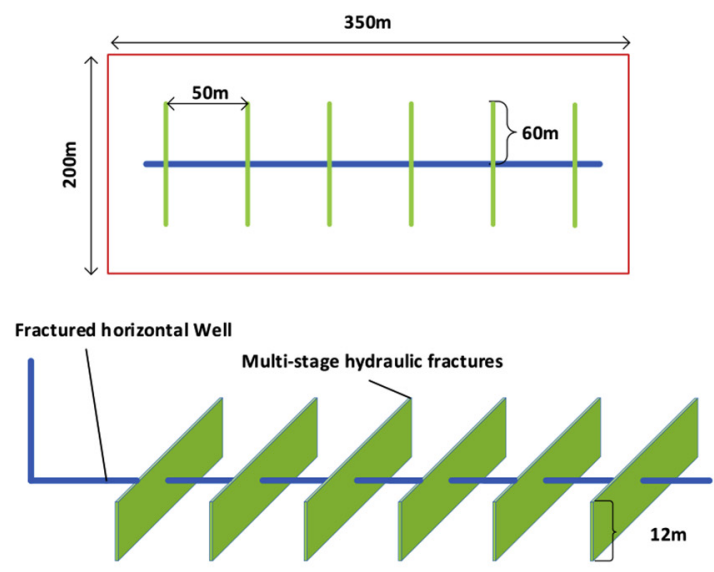

(a)

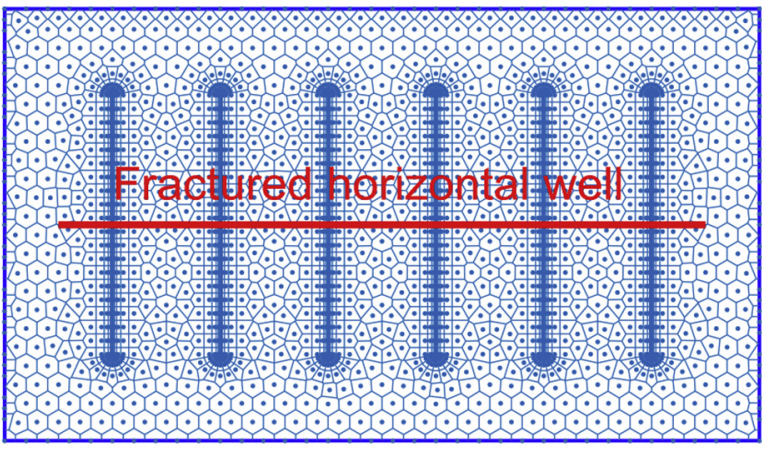

(b)

Fig. B1. (a) Multi-stage hydraulic fracturing reservoir. (b) PEBI grids. In this paper, the hydraulic fractures are modeled as grids and the horizontal wellbore only connects the fractures. The fractures are treated as infinite conductivity.

\section{References}

Akkutlu, I.Y., Efendiev, Y., Savatorova, V., 2015. Multi-scale asymptotic analysis of gas transport in shale matrix. Transp. Porous Media 107 (1), 235-260.

Akkutlu, I.Y., Efendiev, Y., Vasilyeva, M., Wang, Y., 2017. Multiscale model reduction for shale gas transport in a coupled discrete fracture and dual-continuum porous media. J. Nat. Gas Sci. Eng. 48, 65-76.

Akkutlu, I.Y., Fathi, E., 2012. Multiscale gas transport in shales with local kerogen heterogeneities. Spe J. 17 (4), 1002-1011.

Balhoff, M.T., Thomas, S.G., Wheeler, M.F., 2008. Mortar coupling and upscaling of porescale models. Comput. Geosci. 12 (1), 15-27.

Bear, J., 1972. Dynamics of Fluids in Porous media. American Elsevier Pub. Co.

Cao, G., et al., 2017. A 3D coupled model of organic matter and inorganic matrix for calculating the permeability of shale. Fuel 204, 129-143.

Chen, C., 2016. Multiscale imaging, modeling, and principal component analysis of gas transport in shale reservoirs. Fuel 182, 761-770.

Chen, L., et al., 2015a. Heterogeneity of the lower Silurian Longmaxi marine shale in the southeast Sichuan Basin of China. Mar. Petroleum Geol. 65, 232-246.

Chen, Z., Liao, X., Zhao, X., Dou, X., Zhu, L., 2015b. Performance of horizontal wells with fracture networks in shale gas formation. J. Petroleum Sci. Eng. 133, 646-664.

Civan, F., Rai, C.S., Sondergeld, C.H., 2010. Shale-gas permeability and diffusivity inferred by improved formulation of relevant retention and transport mechanisms. Transp. Porous Media 86 (3), 925-944.

Civan, F., Rai, C.S., Sondergeld, C.H., 2011. Shale-gas permeability and diffusivity inferred by improved formulation of relevant retention and transport mechanisms. Transp. Porous Media 86 (3), 925-944.

Costanza-Robinson, M.S., Estabrook, B.D., Fouhey, D.F., 2011. Representative elementary volume estimation for porosity, moisture saturation, and air-water interfacial areas in unsaturated porous media: data quality implications. Water Resour. Res. 47 (7), 12.

Darabi, H., Ettehad, A., Javadpour, F., Sepehrnoori, K., 2012. Gas flow in ultra-tight shale strata. J. Fluid Mech. 710 (12), 641-658.

Geng, L., et al., 2016. A diffusion-viscous flow model for simulating shale gas transport in nano-pores. Fuel 181, 887-894.

Idowu, N.A., Blunt, M.J., 2010. Pore-scale modelling of rate effects in waterflooding Transp. Porous Media 83 (1), 151-169.

Javadpour, F., 2009. Nanopores and apparent permeability of gas flow in mudrocks (shales and siltstone). J. Can. Petroleum Technol. 48 (8), 16-21.

Jiang, W., Lin, M., Yi, Z., Li, H., Wu, S., 2017. Parameter determination using 3D FIB-SEM images for development of effective model of shale gas flow in nanoscale pore clusters. Transp. Porous Media 117 (1), 5-25.

Kelly, S., El-Sobky, H., Torres-Verdín, C., Balhoff, M.T., 2015. Assessing the utility of FIBSEM images for shale digital rock physics. Adv. Water Resour. 95, 302-316.

Klinkenberg, L.J., 1941. The permeability of porous media to liquids and gases. Socar Proc. 2 (2), 200-213.

Lake, L.W., Srinivasan, S., 2004. Statistical scale-up of reservoir properties: concepts and applications. J. Petroleum Sci. Eng. 44 (1-2), 27-39.

Lee, A., Gonzalez, M., Eakin, B., 1966. The viscosity of natural gases. J. Petroleum
Technol. 18 (8), 997-1000.

Liang, D., Guo, T., Chen, J., 2008. Some progresses on studies of hydrocarbon generation and accumulation in marine sedimentary regions, southern China (Part 1): distribution of four suits of regional marine source rocks. Mar. Orig. Pet. Geol. 13, 1-16.

Mahmoud, M.A., 2013. Development of a new correlation of gas compressibility factor (ZFactor) for high pressure gas reservoir. J. Energy Resour. Technol. 136 (1) 2280-2285.

Mehmani, A., Prodanović, M., Javadpour, F., 2013. Multiscale, multiphysics network modeling of shale matrix gas flows. Transp. Porous Media 99 (2), 377-390.

Mehmani, Y., Balhoff, M.T., 2014. Bridging from pore to continuum: a hybrid mortar domain decomposition framework for subsurface flow and transport. Siam J. Multiscale Model. Simul. 12 (2), 667-693.

Naraghi, M.E., Javadpour, F., 2015. A stochastic permeability model for the shale-gas systems. Int. J. Coal Geol. 140, 111-124.

Osiptsov, A.A., Boronin, S.A., Zilonova, E.M., Desroches, J., 2017. Managed SaffmanTaylor instability during overflush in hydraulic fracturing. J. Petroleum Sci. Eng.

Singh, H., 2014. Scale-up of Reactive Processes in Heterogeneous Media.

Singh, H., 2017. Representative elementary volume(REV) in spatio-temporal domain: a method to find REVfor dynamic pores. J. Earth Sci. 28 (2), 391-403.

Singh, H., Cai, J., 2018. Screening improved recovery methods in tight-oil formations by injecting and producing through fractures. Int. J. Heat Mass Transf. 116, 977-993.

Singh, H., Javadpour, F., 2016. Langmuir slip-Langmuir sorption permeability model of shale. Fuel 164, 28-37.

Singh, H., Srinivasan, S., 2014. Scale up of reactive processes in heterogeneous media numerical experiments and semi-analytical modeling. In: SPE Improved Oil Recovery Symposium.

Song, W., et al., 2016. Apparent gas permeability in an organic-rich shale reservoir. Fuel 181, 973-984.

Talonov, A., Vasilyeva, M., 2016. On numerical homogenization of shale gas transport. J. Comput. Appl. Math. 301, 44-52.

Wang, J., Chen, L., Kang, Q., Rahman, S.S., 2016. Permeability prediction of organic shale with generalized lattice Boltzmann model considering surface diffusion effect. Fuel $181,478-490$.

Yan, B., et al., 2018. Multi-porosity multi-physics compositional simulation for gas storage and transport in highly heterogeneous shales. J. Petroleum Sci. Eng. 160, 498-509.

Yang, C., Vyas, A., Datta-Gupta, A., Ley, S.B., Biswas, P., 2017. Rapid multistage hydraulic fracture design and optimization in unconventional reservoirs using a novel fast marching method. J. Petroleum Sci. Eng. 156, 91-101.

Yi, Z.X., et al., 2017. Pore network extraction from pore space images of various porous media systems. Water Resour. Res. 53, 3424-3445.

Zhang, M., et al., 2018. Molecular and carbon isotopic variation in 3.5 years shale gas production from Longmaxi Formation in Sichuan Basin, China. Mar. Petroleum Geol. 89 (Part 1), 27-37.

Zhiming, C., et al., 2014. Productivity estimations for vertically fractured wells with asymmetrical multiple fractures. J. Nat. Gas Sci. Eng. 21, 1048-1060. 